\title{
Analysis of linear general rate model of reactive chromatography for core-shell adsorbents
}

\author{
Shamsul Qamar, ${ }^{*, \dagger}+$ Seemab Bashir, $₫$ Sadia Perveen, $"$ and Andreas \\ Seidel-Morgenstern ${ }^{\dagger}$ \\ $\dagger^{*}$ Max Planck Institute for Dynamics of Complex Technical Systems, Magdeburg, Germany \\ $\ddagger$ COMSATS Institute of Information Technology, Islamabad, Pakistan \\ IAir University, PAF Complex, Sector E-9 Islamabad, Pakistan \\ E-mail: *qamar@mpi-magdeburg.mpg.de
}

\begin{abstract}
This article presents semi-analytical solutions of a linear general rate model for fixed-bed liquid chromatographic reactors packed with core-shell particles. The model considers axial dispersion, interfacial mass transfer, intraparticle diffusion, linear adsorption, heterogeneous irreversible and reversible reactions, and injection of rectangular pulses. The Laplace transformation and eigen-decomposition technique are simultaneously applied to derive analytical solutions. The numerical Laplace inversion is applied for back transforming solutions in the actual time domain. A high resolution finite volume scheme is used to numerically approximate the model equations. Different case studies of reactive chromatography are considered to analyze the effect of core radius fraction on the elution profiles.
\end{abstract}




\section{Introduction}

Core-shell particles or focused-cored beads are formed by an impermeable inner core surrounded by a layer of fully porous silica and, thus, are morphologically quite different from the commonly used fully-porous silica particles. ${ }^{1-5}$ The core may be a single sphere or aggregation of several small spheres. The concept of core-shell particles was introduced by Horvath et al. ${ }^{6}$ at the end of the 1960s to prepare columns that could provide efficient separation of high molecular weight compounds of biological origin in high performance liquid chromatography (HPLC). However, the production of core-shell particles to high-quality standards had to overcome many difficulties in the following decades. ${ }^{7,8}$ Non-porous beads have been a success in analytical liquid chromatography (LC) because they provide sharp peaks and fast separations. ${ }^{9-13}$ However, these beads do not offer sufficient binding sites

for peak separations in preparative LC. ${ }^{1,14-16}$ Fully porous and non-porous beads offer two extreme cases for mass transfer and binding capacities. The first one deals with largest binding capacities and diffusional intraparticle mass transfer resistance, while the second one offers the smallest binding capacities and complete elimination of intraparticle mass transfer. In preparative and large-scale LC, a substantial binding capacity is needed to create retention time differences among peaks. However, with fully porous beads, band broadening makes baseline separation difficult when profiles travel inside the column for sufficiently long time. Thus, core-shell particles offer a compromise between fully porous and non-porous particles that is better for many systems. The performance of separation parameters is also significantly influenced by particle characteristics, such as size of particles and the thickness of porous shell. As the thickness of the porous shell decreases, the mass transfer fasters, the column efficiency improves and the retention time reduces. The size of the particle core, the shell porosity and the thickness of the shell are adjusted accordingly to suit distinct chromatographic applications. Core-shell columns are now commercially 
available in particle sizes ranging from 1.3 to $5 \mu \mathrm{m}$. Usually, columns packed with $2.7 \mu \mathrm{m}$ core-shell particles will produce efficiencies approaching those packed with $1.8 \mu \mathrm{m}$ fully porous particles, but at significantly lower back pressures. In HPLC applications, the core shell silica particles with a solid core and porous shell are commonly used. Ding and Sun ${ }^{17}$ fabricated gel-coated cored beads with dense magnetic cores for protein separations in a magnetically stabilized fluidized bed. The use of a rigid core relieves bed compression for soft gels and, thus, during scale-up, cored beads allow longer columns that are better for peaks resolution. ${ }^{18}$ Cored beads were also used in gas chromatography. ${ }^{19}$

Cored beads have been used in various LC separations, especially in ion exchange. ${ }^{6,18,20,21}$ They are also practically used for the separations of proteins and peptides, ${ }^{1,4,22}$ including proteins in proteomic research, ${ }^{18}$ nucleotides, ${ }^{6}$ and other compounds. ${ }^{21}$ The authors in ${ }^{23,24}$ performed experiments to compare and analyze several commercially available fully porous and cored beads. Zhou et al. ${ }^{4}$ considered a general rate model for cored beads to obtain intraparticle diffusion coefficients. They did not provide numerical methods or simulated elusion profiles. Kaczmarski and Guiochon ${ }^{25,26}$ extended the standard models of chromatography to columns packed with core-shell particles. Li et al. ${ }^{27}$ performed optimization of core size for linear chromatography by minimizing height equivalent theoretical plate (HETP) number. Lambert et al. ${ }^{28}$ have studied the mass-transfer properties of insulin on core shell and fully porous particles. Recently, we have analyzed linear and nonlinear models for liquid chromatographic columns packed with core-shell particles..$^{29,30}$ Reactive chromatography is an integrated process in which chemical or biochemical reactions are combined with the chromatographic separation. ${ }^{31-34}$ This technique improves the conversion of reactants and purity of products and, therefore, remained a source of attraction to several researchers in the past few decades. ${ }^{31,33,35-50}$ The chemical reactions can be of two types, such as homogeneous and heterogeneous. For understanding the basic principle of a fixed-bed chromatographic reactor, let us consider a single column reactor 
and a reversible reaction of the type $\mathrm{A} \rightleftarrows \mathrm{B}$. The reactant $A$ is dissolved in the desorbent and is injected as rectangular pulse into the column packed with the stationary phase. The reaction occurs at the surface of catalyst to form the product $B$. Both components $A$ and $B$ interact with the surface of adsorbent and because of their different affinities to the stationary phase, they move inside the column with different propagation speeds. Hence, components are separated and the driving force for the forward reaction is enhanced and the backward reaction is suppressed. As a result, chemical equilibrium can be achieved and high purity product can be obtained at the column outlet. Recently, we have theoretically studied reactive liquid chromatopathy by considering different linear and nonlinear chromatographic models. ${ }^{51-56}$

This paper is concerned with the analytical solutions of a two-component linear general rate model (GRM) for fixed-bed liquid chromatographic reactors packed with a spherical shaped core-shell particles. The model considers two-component mixture, irreversible and reversible reactions, axial dispersion, interfacial mass transfer, intraparticle diffusion, linear adsorption, and rectangular pulse injections. It is an extension of our recent works on reactive chromatography considering fully porous particles. ${ }^{55}$ Analytical solutions of the model equations are derived by simultaneously applying the Laplace transformation and eigen-decomposition techniques. ${ }^{57,58}$ The numerical Laplace inversion is applied for back transformation of the solutions in the actual time domain. ${ }^{59}$ Numerical simulations are required to accurately predict the dynamic behavior of chromatographic columns. To gain further confidence on the derived semi-analytical solutions, a high resolution finite volume scheme (HR-FVS) is applied to solve the model equations numerically. ${ }^{60,61}$ Different case studies of the two-component mixtures are considered. With these case studies it is intended to analyze the effects of core radius fraction, reaction rate constant, axial dispersion, film mass transfer resistance and intraparticle diffusion resistance, on the elution curves. The behavior of chromatographic reactor is further analyzed by calculating 
numerical moments of concentration profile.

This article is structured as follows. In Section 2, the two-component linear reactive GRM for core-shell particles is introduced for irreversible reaction along with two sets of boundary conditions (BCs), such as Dirichlet and Danckwerts BCs. In Section 3, analytical solutions are derived to solve these model equations for the considered irreversible reaction. In Section 4, the model and analysis are extended for the case of reversible reaction. In Section 5, several test problems are considered. Finally, conclusions are presented in Section 6.

\section{Irreversible reaction $(\mathrm{A} \rightarrow \mathrm{B})$ in a fixed-bed chromato-}

\section{graphic reactor}

This study considers an isothermal chromatographic reactor packed with core-shell particles, see Figure 1. Concentration pulses of the reactant are periodically injected to a flowing stream passing through the chromatographic reactor. In this process, the reactant $A$ (component 1 ) converts into product $B$ (component 2) through an irreversible first order heterogeneous reaction. The considered GRM contains four mass balance equations for describing concentrations transport in the bulk of fluid and inside the particles micropores. The mass balance equations in the bulk of fluid are expressed as $^{31,32}$

$$
\frac{\partial c_{b_{i}}}{\partial t}+u \frac{\partial c_{b_{i}}}{\partial z}=D_{b} \frac{\partial^{2} c_{b_{i}}}{\partial z^{2}}-\frac{3}{R_{p}} F_{b} k_{\mathrm{ext}_{i}}\left(c_{b_{i}}-\left.c_{p_{i}}\right|_{r=R_{p}}\right), \quad i=1,2 .
$$

Here, $t$ denotes the time coordinate, $z$ represents the axial coordinate along the column length, $c_{b_{i}}(t, z)$ is the concentration of the $i$ th component in the bulk of fluid and $c_{p_{i}}(t, z)$ is the concentration of $i$ th component in the particle pores, respectively. Moreover, $u$ is the interstitial velocity, $D_{b}$ represents the axial dispersion coefficient which quantifies back

mixing effects in the column and is assumed same for both components, $k_{\text {ext }, i}$ is the external 
mass transfer coefficient of $i$ th component quantifying the rate of the transport through a luminary boundary layer, $F_{b}=\frac{1-\epsilon_{b}}{\epsilon_{b}}$ is the phase ratio, $\epsilon_{b} \in(0,1)$ is the external porosity, and $r$ denotes the radial coordinate of spherical particles of radius $R_{p}$ (c.f. Figure 1 ). Assuming pore-diffusion as a mean of intraparticle transport, the governing equations in the particles pores are given as: ${ }^{31,32}$

$$
\epsilon_{p} \frac{\partial c_{p_{i}}}{\partial t}+\left(1-\epsilon_{p}\right) \frac{\partial q_{p_{i}}^{*}}{\partial t}=\frac{1}{r^{2}} \frac{\partial}{\partial r}\left(r^{2}\left[\epsilon_{p} D_{p_{i}} \frac{\partial c_{p_{i}}}{\partial r}+\left(1-\epsilon_{p}\right) D_{s_{i}} \frac{\partial q_{p_{i}}^{*}}{\partial r}\right]\right) \mp \nu_{1}\left(1-\epsilon_{p}\right) q_{p_{1}}^{*}
$$

Here, $i=1,2$. In the above equation, $q_{p_{i}}^{*}$ denotes the local equilibrium concentration of $i$ th component in the stationary phase, $\epsilon_{p}$ is the internal porosity, $D_{p_{i}}$ is the pore diffusivity of $i$ th component, $D_{s_{i}}$ is the surface diffusivity of $i$ th component, and $\nu_{1}$ is the rate constant of the chemical reaction taking place in the column. The signs of the reaction rate constant $\nu_{1}$ is taken positive for component 1 and negative for component 2 indicating the irreversible behavior of reactant (component 1).

The Eqs. (1)-(2) are linked at $r=R_{p}$ through the following relations: ${ }^{32}$

$$
\left[\epsilon_{p} D_{p_{i}} \frac{\partial c_{p_{i}}}{\partial r}+\left(1-\epsilon_{p}\right) D_{s_{i}} \frac{\partial q_{p_{i}}^{*}}{\partial r}\right]_{r=R_{p}}=k_{\text {ext }_{i}}\left(c_{b_{i}}-\left.c_{p_{i}}\right|_{r=R_{p}}\right), \quad i=1,2 .
$$

The adsorption isotherms are assumed linear in this study, ${ }^{32}$ i.e.

$$
q_{p_{i}}^{*}=a_{i} c_{p_{i}}, \quad i=1,2
$$

After plugging Eq. (4) in Eq. (2), we get

$$
a_{i}^{*} \frac{\partial c_{p_{i}}}{\partial t}=\frac{D_{\mathrm{eff}_{i}}}{r^{2}} \frac{\partial}{\partial r}\left(r^{2} \frac{\partial c_{p_{i}}}{\partial r}\right) \mp \nu_{1}\left(1-\epsilon_{p}\right) a_{1} c_{p_{1}}
$$

with a component specific effective diffusivity $D_{\text {eff }_{i}}$ coefficient quantifying the transport 
rate in the particles:

$$
D_{\mathrm{eff}_{i}}=\epsilon_{p} D_{p_{i}}+\left(1-\epsilon_{p}\right) D_{s_{i}} a_{i}, \quad a_{i}^{*}=\epsilon_{p}+\left(1-\epsilon_{p}\right) a_{i}, \quad i=1,2
$$

Similarly, Eq. (3) reduces to

$$
\left.D_{\text {eff }_{i}} \frac{\partial c_{p_{i}}}{\partial r}\right|_{r=R_{p}}=k_{\operatorname{ext}_{i}}\left(c_{b_{i}}-\left.c_{p_{i}}\right|_{r=R_{p}}\right) .
$$

The following dimensionless variables are introduced for simplifying the analysis and for reducing the number of variables:

$$
\begin{aligned}
& x=\frac{z}{L}, \quad \tau=\frac{u t}{L}, \quad \rho=\frac{r}{R_{p}}, \quad P e_{b}=\frac{L u}{D_{b}}, \quad \omega_{1}=\frac{\nu_{1} L}{u}, \\
& B i_{p_{i}}=\frac{k_{\mathrm{ext}_{i}} R_{p}}{D_{\mathrm{eff}_{i}}}, \quad \eta_{p_{i}}=\frac{D_{\mathrm{eff}_{i} L} L}{R_{p}^{2} u}, \quad \xi_{p_{i}}=3 B i_{p_{i}} \eta_{p_{i}} F_{b}, \quad i=1,2,
\end{aligned}
$$

where $L$ denotes the column length, $P e_{b}$ represents the axial Pectlet number which is assumed same for both components, and $\omega_{1}$ quantifies the reaction rate. By introducing the dimensionless variables in Eqs. (1), (5) and (7), we get

$$
\begin{aligned}
\frac{\partial c_{b_{i}}}{\partial \tau}+\frac{\partial c_{b_{i}}}{\partial x} & =\frac{1}{P e_{b}} \frac{\partial^{2} c_{b_{i}}}{\partial x^{2}}-\xi_{p_{i}}\left(c_{b_{i}}-\left.c_{p_{i}}\right|_{\rho=1}\right), \\
a_{i}^{*} \frac{\partial c_{p_{i}}}{\partial \tau} & =\frac{\eta_{p_{i}}}{\rho^{2}} \frac{\partial}{\partial \rho}\left(\rho^{2} \frac{\partial c_{p_{i}}}{\partial \rho}\right) \mp \omega_{1}\left(1-\epsilon_{p}\right) a_{1} c_{p_{1}}, \\
\left.\frac{\partial c_{p_{i}}}{\partial \rho}\right|_{\rho=1} & =B i_{p_{i}}\left(c_{b_{i}}-\left.c_{p_{i}}\right|_{\rho=1}\right), \quad i=1,2 .
\end{aligned}
$$

For fully porous particles $\rho$ ranges from 0 to 1 , while for cored particles it ranges from $\beta=R_{\text {core }} / R_{p}$ to 1 . As this study is concerned with the cored particles of arbitrary core radius fraction $\beta$, it is necessary to allow the core radius to be changed. For cored particles (c.f. Eqs. (10) and (11) ), $\beta \leq \rho \leq 1$. For fully porous particles $\beta=0$, while $\beta \neq 0$ for 
cored particles. Thus, it is helpful to replace $\rho$-axis by $0 \leq \gamma \leq 1$, where

$$
\gamma=\frac{\rho-\beta}{1-\beta} .
$$

On substituting

$$
\rho=\gamma(1-\beta)+\beta,
$$

in Eqs. (9)-(11), we obtain

$$
\begin{aligned}
& \frac{\partial c_{b_{i}}}{\partial \tau}+\frac{\partial c_{b_{i}}}{\partial x}=\frac{1}{P e_{b}} \frac{\partial^{2} c_{b_{i}}}{\partial x^{2}}-\xi_{p_{i}}\left(c_{b_{i}}-\left.c_{p_{i}}\right|_{\rho=1}\right) \\
& a_{i}^{*} \frac{\partial c_{p_{i}}}{\partial \tau}-\eta_{b_{i}}\left(\frac{1}{(1-\beta)^{2}} \frac{\partial^{2} c_{p_{i}}}{\partial \gamma^{2}}+\frac{2}{(\gamma(1-\beta)+\beta)} \frac{1}{(1-\beta)} \frac{\partial c_{p_{i}}}{\partial \gamma}\right) \pm \omega_{1}\left(1-\epsilon_{p}\right) a_{1} c_{p_{1}}=0 \\
& \left.\frac{\partial c_{p_{i}}}{\partial \gamma}\right|_{\gamma=1}=B_{p_{i}}(1-\beta)\left(c_{b_{i}}-\left.c_{p_{i}}\right|_{\gamma=1}\right), \quad i=1,2
\end{aligned}
$$

Eqs. (15) can now be rephrased as

$$
\begin{aligned}
& (1-\beta)^{2} a_{i}^{*} \frac{\partial}{\partial \tau}\left[(\gamma(1-\beta)+\beta) c_{p_{i}}\right]-\eta_{b_{i}} \frac{\partial^{2}}{\partial \gamma^{2}}\left[(\gamma(1-\beta)+\beta) c_{p_{i}}\right] \\
& \pm(1-\beta)^{2} \omega_{1}\left(1-\epsilon_{p}\right) a_{1}\left[(\gamma(1-\beta)+\beta) c_{p_{1}}\right]=0, \quad i=1,2 .
\end{aligned}
$$

Moreover, appropriate inlet and outlet BCs are needed for Eqs. (14) and (17). For an initially regenerated column, the corresponding initial conditions are given as

$$
c_{b_{i}}(0, x)=0, \quad c_{p_{i}}(0, x, \gamma)=0, \quad \forall x, \gamma \in(0,1), \quad i=1,2 .
$$


The following boundary conditions at $\gamma=0$ and $\gamma=1$ are assumed for Eq. (17)

$$
\left.\frac{\partial c_{p_{i}}}{\partial \gamma}\right|_{\gamma=0}=0,\left.\quad \frac{\partial c_{p_{i}}}{\partial \gamma}\right|_{\gamma=1}=B_{p_{i}}(1-\beta)\left(c_{b_{i}}-\left.c_{p_{i}}\right|_{\gamma=1}\right), \quad i=1,2 .
$$

Due to assumed rapid adsorption or desorption rates, the concentrations of solute in the pores and that in the stationary phase are in the equilibrium state. In this study, the following two sets of BCs are considered as summarized below.

\section{Type 1: Dirichlet inlet BCs}

In this case, the simpler Dirichlet BCs are applied at the column inlet

$$
c_{b_{i}}(\tau, x=0)=\left\{\begin{array}{ll}
c_{b_{i}}, \text { inj }, & \text { if } \quad 0 \leq \tau \leq \tau_{\text {inj }}, \\
0, & \tau>\tau_{\text {inj }},
\end{array}, i=1,2,\right.
$$

together with the useful and realistic zero Neumann BCs for hypothetically infinite length column (Neumann at infinity):

$$
\left.\frac{\partial c_{b_{i}}}{\partial x}\right|_{x=\infty}=0, \quad i=1,2
$$

This assumption is required to satisfy the over all mass balance i.e mass injected at the column inlet should be equal to the mass at the column outlet. For sufficiently small dispersion coefficient, for example $D_{b} \leq 10^{-5} \mathrm{~m}^{2} / \mathrm{s}$, the Dirichlet BCs are well applicable.

\section{Type 2: Danckwerts inlet BCs}

In this case, the Robin type BCs, also known as Danckwerts BCs in chemical engineering, are applied at the column inlet: ${ }^{62}$

$$
c_{b_{i}}(\tau, x=0)-\frac{1}{P e_{b}} \frac{\partial c_{b_{i}}(\tau, x=0)}{\partial x}=\left\{\begin{array}{cc}
c_{b_{i}} \text {,inj }, & \text { if } \quad 0 \leq \tau \leq \tau_{\text {inj }}, \\
0, & \tau>\tau_{\text {inj }},
\end{array}, \quad i=1,2 .\right.
$$


Danckwert BCs quantify the possible back mixing at the column inlet due to large axial dispersion and reduces to simpler Dirichlet BCs when the axial dispersion is small. At the outlet of the column of finite length $L$, the following Neumann BCs are used

$$
\left.\frac{\partial c_{b_{i}}}{\partial x}\right|_{x=1}=0 .
$$

\section{Analytical solutions of the model for reaction of type}

\section{$\mathrm{A} \rightarrow \mathrm{B}$}

Here, semi-analytical solutions of the above model are presented for Dirichlet (Eqs. (20a) and (20b)) and Danckwert (Eqs. (21a) and (21b)) inlet boundary conditions. The following Laplace transformation is applied as a basic tool to solve the model equations:

$$
\bar{c}_{i}(s, x)=\int_{0}^{\infty} e^{-s \tau} c_{i}(\tau, x) d \tau, \quad \tau \geq 0, \quad c \in\left\{c_{b}, c_{p}\right\} .
$$

After applying the Laplace transformation, the model Eqs. (14) yield

$$
s \bar{c}_{b_{i}}+\frac{d \bar{c}_{b_{i}}}{d x}=\frac{1}{P e_{b}} \frac{d^{2} \bar{c}_{b_{i}}}{d x^{2}}-\xi_{p_{i}}\left(\bar{c}_{b_{i}}-\left.\bar{c}_{p_{i}}\right|_{\gamma=1}\right), \quad i=1,2 .
$$

While, the Laplace transformation of Eqs. (17) gives

$$
\begin{aligned}
& (1-\beta)^{2} a_{i}^{*}\left[(\gamma(1-\beta)+\beta) \bar{c}_{p_{i}}\right] s-\eta_{p_{i}} \frac{d^{2}}{d \gamma^{2}}\left[(\gamma(1-\beta)+\beta) \bar{c}_{p_{i}}\right] \\
& \pm(1-\beta)^{2} \omega_{1}\left(1-\epsilon_{p}\right) a_{1}\left[(\gamma(1-\beta)+\beta) \bar{c}_{p_{1}}\right]=0, \quad i=1,2 .
\end{aligned}
$$


The general solution of Eq. (24) for $(i=1)$ is given as

$$
\bar{c}_{p_{1}}(s, x, \gamma)=\frac{1}{(\gamma(1-\beta)+\beta)}\left[k_{1} e^{(1-\beta) \sqrt{\alpha(s)} \gamma}+k_{2} e^{-(1-\beta) \sqrt{\alpha(s)} \gamma}\right]
$$

where,

$$
\alpha(s)=\frac{a_{1}^{*} s+\omega_{1}\left(1-\epsilon_{p}\right) a_{1}}{\eta_{p_{1}}} .
$$

On utilizing the boundary conditions given in Eq. (19), the values of $k_{1}$ and $k_{2}$ become

$$
\begin{aligned}
& k_{1}=\frac{B i_{p_{1}} \bar{c}_{b_{1}}(\beta(\sqrt{\alpha(s)})+1) / 2 \sinh ((1-\beta) \sqrt{\alpha(s)})}{\left((1-\beta)+B i_{p_{1}} \beta\right) \sqrt{\alpha(s)}\left[\frac{\left(B i_{p_{1}}+(\beta \alpha(s)-1)\right)}{\left((1-\beta)+B i_{p_{1}} \beta\right) \sqrt{\alpha(s)}}+\operatorname{coth}((1-\beta) \sqrt{\alpha(s)})\right]}, \\
& k_{2}=\left[\frac{(\beta(\sqrt{\alpha(s)})-1)}{(\beta(\sqrt{\alpha(s)})+1)}\right] \frac{B i_{p_{1}} \bar{c}_{b_{1}}(\beta(\sqrt{\alpha(s)})+1) / 2 \sinh ((1-\beta) \sqrt{\alpha(s)})}{\left((1-\beta)+B i_{p_{1}} \beta\right) \sqrt{\alpha(s)}\left[\frac{\left(B i_{p_{1}}+(\beta \alpha(s)-1)\right)}{\left((1-\beta)+B i_{p_{1}} \beta\right) \sqrt{\alpha(s)}}+\operatorname{coth}((1-\beta) \sqrt{\alpha(s)})\right]} .
\end{aligned}
$$

Here, the upper positive sign is taken for $k_{1}$ and the lower negative sign for $k_{2}$. At $\gamma=1$, Eqs. (25), (27) and (28) give

$$
\left.\bar{c}_{p_{1}}\right|_{\rho=1}=\bar{c}_{b_{1}} f_{1}(s),
$$

where

$$
f_{1}(s)=\frac{B i_{p_{1}}[\beta(\sqrt{\alpha(s)}) \operatorname{coth}((1-\beta) \sqrt{\alpha(s)})+1]}{\left((1-\beta)+B i_{p_{1}} \beta\right) \sqrt{\alpha(s)}\left[\frac{\left(B i_{p_{1}}+(\beta \alpha(s)-1)\right)}{\left((1-\beta)+B i_{p_{1}} \beta\right) \sqrt{\alpha(s)}}+\operatorname{coth}((1-\beta) \sqrt{\alpha(s)})\right]} .
$$


Introducing Eq. (21b) in Eq. (18), we get the general solution

$$
\begin{aligned}
& \bar{c}_{p_{2}}(s, x, \rho)=\frac{1}{(\gamma(1-\beta)+\beta)}\left[k_{1}^{\prime} e^{(1-\beta) \sqrt{\alpha^{\prime}(s)} \gamma}+k_{2}^{\prime} e^{-(1-\beta) \sqrt{\alpha^{\prime}(s)} \gamma}\right] \\
& -\frac{f_{1}(s)\left(1-\epsilon_{p}\right) a_{1} \omega \bar{c}_{b_{1}}(\beta(\sqrt{\alpha(s)}) \cosh ((1-\beta) \sqrt{\alpha(s) \gamma}+\sinh ((1-\beta) \sqrt{\alpha(s) \gamma}))}{(\gamma(1-\beta)+\beta)\left(\alpha(s) \eta_{p_{2}}-a_{2}^{*} s\right)(\beta \sqrt{\alpha(s)}-1)(\beta(\sqrt{\alpha(s)}) \operatorname{coth}((1-\beta) \sqrt{\alpha(s) \gamma}+1)(\sinh (1-\beta) \sqrt{\alpha(s)})}
\end{aligned}
$$

where $\alpha^{\prime}(s)=\frac{a_{2}^{*} s}{\eta_{p_{2}}}$. By using the boundary conditions in Eq. (19) in Eq. (31), we get

$k_{1}^{\prime}=\frac{B i_{p_{2}} \bar{c}_{b_{2}}+\frac{\left(\left(B i_{p_{2}}-1\right)+\sqrt{\alpha(s)}(\beta(\sqrt{\alpha(s)}) \operatorname{coth}((1-\beta) \sqrt{\alpha(s)}))\right) f_{1}(s)\left(1-\epsilon_{p}\right) a_{1} \omega \bar{c}_{b_{1}}}{\left(\alpha(s) \eta_{p_{2}}-a_{2}^{*} s\right)(\beta \sqrt{\alpha(s)}-1)}}{2 \sinh \left((1-\beta) \sqrt{\alpha^{\prime}(s)}\right)\left[-1+\beta \alpha^{\prime}(s)+B i_{p_{2}}+\left(1-\beta+\beta B i_{p_{2}}\right) \sqrt{\alpha^{\prime}(s)} \operatorname{coth}\left((1-\beta) \sqrt{\alpha^{\prime}(s)}\right)\right]}$,

$$
\begin{aligned}
k_{2}^{\prime}= & {\left[\frac{\left(\beta\left(\sqrt{\alpha^{\prime}(s)}\right)-1\right)}{\left(\beta\left(\sqrt{\alpha^{\prime}(s)}\right)+1\right)}\right] } \\
& \frac{B i_{p_{2}} \bar{c}_{b_{2}}+\frac{\left(\left(B i_{p_{2}}-1\right)+\sqrt{\alpha(s)}(\beta(\sqrt{\alpha(s)}) \operatorname{coth}((1-\beta) \sqrt{\alpha(s)}))\right) f_{1}(s)\left(1-\epsilon_{p}\right) a_{1} \omega \bar{c}_{b_{1}}}{\left(\alpha(s) \eta_{p_{2}}-a_{2}^{*} s\right)(\beta \sqrt{\alpha(s)}-1)}}{2 \sinh \left((1-\beta) \sqrt{\alpha^{\prime}(s)}\right)\left[-1+\beta \alpha^{\prime}(s)+B i_{p_{2}}+\left(1-\beta+\beta B i_{p_{2}}\right) \sqrt{\alpha^{\prime}(s)} \operatorname{coth}\left((1-\beta) \sqrt{\alpha^{\prime}(s)}\right)\right]} .
\end{aligned}
$$

At $\gamma=1$, Eqs. (31), (32) and (33) give

$$
\left.\bar{c}_{p_{2}}\right|_{\gamma=1}=\bar{c}_{b_{2}} f_{2}(s)+\bar{c}_{b_{1}} A(s)
$$

where,

$$
f_{2}(s)=\frac{B i_{p_{2}}\left[\beta\left(\sqrt{\alpha^{\prime}(s)}\right) \operatorname{coth}\left((1-\beta) \sqrt{\alpha^{\prime}(s)}\right)+1\right]}{\left((1-\beta)+B i_{p_{2}} \beta\right) \sqrt{\alpha^{\prime}(s)}\left[\frac{\left(B i_{p_{2}}+\left(\beta \alpha^{\prime}(s)-1\right)\right)}{\left((1-\beta)+B i_{p_{2}} \beta\right) \sqrt{\alpha^{\prime}(s)}}+\operatorname{coth}\left((1-\beta) \sqrt{\alpha^{\prime}(s)}\right)\right]},
$$


and

$$
\begin{aligned}
A(s)= & \frac{f_{1}(s)\left(1-\epsilon_{p}\right) a_{1} \omega}{\left(\alpha(s) \eta_{p_{2}}-a_{2}^{*} s\right)(\beta \sqrt{\alpha(s)}-1)} \\
& {\left[\frac{\left(\left(B i_{p_{2}}-1\right)+\sqrt{\alpha(s)}(\beta \sqrt{\alpha(s)}+\operatorname{coth}((1-\beta) \sqrt{\alpha(s)}))\left[\beta\left(\sqrt{\alpha^{\prime}(s)}\right) \operatorname{coth}\left((1-\beta) \sqrt{\alpha^{\prime}(s)}\right)+1\right]\right.}{\left((1-\beta)+B i_{p_{2}} \beta\right) \sqrt{\alpha^{\prime}(s)}\left[\frac{\left(B i_{p_{2}}+\left(\beta \alpha^{\prime}(s)-1\right)\right)}{\left((1-\beta)+B i_{p_{2}} \beta\right) \sqrt{\alpha^{\prime}(s)}}+\operatorname{coth}\left((1-\beta) \sqrt{\alpha^{\prime}(s)}\right)\right]} .\right.}
\end{aligned}
$$

On introducing Eqs. (29) and (34) in Eqs. (23) for $(i=1,2)$, we get the following ordinary differential equations (ODEs)

$$
\begin{gathered}
\frac{d^{2} \bar{c}_{b_{1}}}{d x^{2}}-P e_{b} \frac{d \bar{c}_{b_{1}}}{d x}-P e_{b} \phi_{1}(s) \bar{c}_{b_{1}}=0 \\
\frac{d^{2} \bar{c}_{b_{2}}}{d x^{2}}-P e_{b} \frac{d \bar{c}_{b_{2}}}{d x}-P e_{b} \phi_{2}(s) \frac{d \bar{c}_{b_{2}}}{d x}=-P e_{b} \xi_{p_{2}} A(s) \bar{c}_{b_{1}},
\end{gathered}
$$

where

$$
\phi_{1}(s)=s+\xi_{p_{1}}\left(1-f_{1}(s)\right), \quad \phi_{2}(s)=s+\xi_{p_{2}}\left(1-f_{2}(s)\right) .
$$

Eqs. (37) and (38) can be expressed in matrix notation as

$$
\frac{d^{2}}{d x^{2}}\left(\begin{array}{c}
\bar{c}_{b_{1}} \\
\bar{c}_{b_{2}}
\end{array}\right)-P e_{b} \frac{d}{d x}\left(\begin{array}{c}
\bar{c}_{b_{1}} \\
\bar{c}_{b_{2}}
\end{array}\right)-\left[\begin{array}{cc}
P e_{b} \phi_{1}(s) & 0 \\
-\xi_{p_{2}} P e_{b} A(s) & P e_{b} \phi_{2}(s)
\end{array}\right]\left(\begin{array}{l}
\bar{c}_{b_{1}} \\
\bar{c}_{b_{2}}
\end{array}\right)=\left(\begin{array}{l}
0 \\
0
\end{array}\right)
$$

Here, the square brackets [ ] stands for a square matrix, the round brackets ( ) represents a column vector, and $\bar{c}_{b_{i}}$ for $(i=1,2)$ are the liquid phase concentrations of mixture components in the Laplace domain. 
The reaction coefficient matrix $[B]$ in Eq. (40) is given as

$$
B=\left[\begin{array}{cc}
P e_{b} \phi_{1}(s) & 0 \\
-\xi_{p_{2}} P e_{b} A(s) & P e_{b} \phi_{2}(s)
\end{array}\right]
$$

In the next step, the linear transformation matrix $[A]$ will be computed. ${ }^{52,53,58}$ Note that, the columns of $[A]$ are the eigenvectors of the matrix $[B]$. The eigenvalues and eigenvectors of the matrix $[B]$ are given as:

$$
\lambda_{1}=P e_{b} \phi_{1}(s), \quad x_{1}=\left(\begin{array}{c}
A_{11} \\
\frac{-\xi_{p_{2}} P e_{b} A(s) A_{11}}{P e_{b} \phi_{1}(s)-P e_{b} \phi_{2}(s)}
\end{array}\right) \quad \text { and } \lambda_{2}=P e_{b} \phi_{2}(s), \quad x_{2}=\left(\begin{array}{c}
0 \\
A_{22}
\end{array}\right) .
$$

Here, $\lambda_{1}$ and $\lambda_{2}$ denote the eigenvalues and $A_{11}$ and $A_{22}$ are the arbitrary constants. For simplicity, we take the values of $A_{11}$ and $A_{22}$ equal to one. Using Eq. (42), the diagonal matrix $\widetilde{k}$ and the transformation matrix $[A]$ can be written as

$$
\widetilde{k}=\left[\begin{array}{cc}
P e_{b} \phi_{1}(s) & 0 \\
0 & P e_{b} \phi_{2}(s)
\end{array}\right], \quad A=\left[\begin{array}{cc}
1 & 0 \\
\frac{-\xi_{p_{2}} P e_{b} A(s)}{P e_{b} \phi_{1}(s)-P e_{b} \phi_{2}(s)} & 1
\end{array}\right] .
$$

The matrix $[A]$ is then used in the following linear transformation ${ }^{53,58}$

$$
\left(\begin{array}{c}
\bar{c}_{b_{1}} \\
\bar{c}_{b_{2}}
\end{array}\right)=\left[\begin{array}{cc}
1 & 0 \\
\frac{-\xi_{p_{2}} P e_{b} A(s)}{P e_{b} \phi_{1}(s)-P e_{b} \phi_{2}(s)} & 1
\end{array}\right]\left(\begin{array}{l}
b_{1} \\
b_{2}
\end{array}\right)
$$

Applying the above linear transformation on Eq. (40), we get

$$
\frac{d^{2}}{d x^{2}}\left(\begin{array}{l}
b_{1} \\
b_{2}
\end{array}\right)-P e_{b} \frac{d}{d x}\left(\begin{array}{l}
b_{1} \\
b_{2}
\end{array}\right)=\left[\begin{array}{cc}
P e_{b} \phi_{1}(s) & 0 \\
0 & P e_{b} \phi_{2}(s)
\end{array}\right]\left(\begin{array}{l}
b_{1} \\
b_{2}
\end{array}\right)
$$


Eq. (45) represents a system of two independent ODEs. Their explicit solutions are given as

$$
b_{1}(s, x)=A_{1} e^{m_{1} x}+B_{1} e^{m_{2} x}, \quad m_{1,2}=\frac{P e_{b}}{2}\left(1 \mp \sqrt{1+\frac{4 \phi_{1}(s)}{P e_{b}}}\right),
$$

and

$$
b_{2}(s, x)=A_{2} e^{m_{3} x}+B_{2} e^{m_{4} x}, \quad m_{3,4}=\frac{P e_{b}}{2}\left(1 \mp \sqrt{1+\frac{4 \phi_{2}(s)}{P e_{b}}}\right) .
$$

Here, $A_{1}, B_{1}, A_{2}$ and $B_{2}$ are constants of integration which can be obtained by using one of the selected two sets of BCs. If $\omega_{1}=0$, the above solutions correctly reduce to the solutions of non-reactive chromatography presented in our previous article. ${ }^{29}$

\section{Type 1: Dirichlet BCs}

The Laplace Transformation of Eqs. (20a) and (20b) gives

$$
\bar{c}_{b_{i}}(s, 0)=\frac{c_{b_{i}, \mathrm{inj}}\left(1-e^{-s \tau_{\mathrm{inj}}}\right)}{s}, \quad \frac{d \bar{c}_{b_{i}}}{d x}(s, \infty)=0 .
$$

On using the transformations in Eq. (44), Eq. (48) yields

$$
\begin{aligned}
& b_{1}(s, x=0)=\frac{\left(1-e^{-s \tau_{\mathrm{inj}}}\right)}{s} c_{b_{1}, \mathrm{inj}}, \quad \frac{d b_{1}}{d x}(s, \infty)=0 . \\
& b_{2}(s, x=0)=\frac{\left(1-e^{-s \tau_{\mathrm{inj}}}\right)}{s} c_{b_{2}, \mathrm{inj}}+\frac{\xi_{p_{2}} P e_{b} A(s)}{P e_{b} \phi_{1}(s)-P e_{b} \phi_{2}(s)} b_{1}(s, x=0), \frac{d b_{2}}{d x}(s, \infty)=0 .
\end{aligned}
$$


After applying these boundary conditions on Eq. (46), the values of $A_{1}$ and $B_{1}$ are obtained as

$$
A_{1}=\frac{\left(1-e^{-s \tau_{\mathrm{inj}}}\right)}{s} c_{b_{1}, \mathrm{inj}}, \quad B_{1}=0 .
$$

Thus, Eqs. (44), Eq. (46) and Eq. (51) gives

$$
\bar{c}_{b_{1}}(s, x)=\frac{c_{b_{1}, \mathrm{inj}}\left(1-e^{-s \tau_{\mathrm{inj}}}\right)}{s} e^{m_{1} x}
$$

where the value of $m_{1}$ is given by Eq. (46) for the upper negative sign. Similarly, on using Eq. (50) in Eq. (47), we get the values of $A_{2}$ and $B_{2}$ as follows:

$$
A_{2}=\frac{\left(1-e^{\left.-s \tau_{\mathrm{inj}}\right)}\right.}{s}\left[c_{b_{2}, \text { inj }}+\frac{\xi_{p_{2}} P e_{b} A(s)}{P e_{b} \phi_{1}(s)-P e_{b} \phi_{2}(s)} c_{b_{1}, \mathrm{inj}}\right], \quad B_{2}=0 .
$$

With these values of $A_{2}$ and $B_{2}$ and using Eq. (44) in Eq. (47), we obtain

$$
\overline{c_{b_{2}}}(s, x)=\frac{c_{b_{1}, \mathrm{inj}}\left(1-e^{-s \tau_{\mathrm{inj}}}\right)}{s}\left(\frac{\xi_{p_{2}} A(s)}{\phi_{1}(s)-\phi_{2}(s)}\right)\left(e^{m_{3} x}-e^{m_{1} x}\right)+\frac{c_{b_{2}, \mathrm{inj}}\left(1-e^{-s \tau_{\mathrm{inj}}}\right)}{s} e^{m_{3} x} .
$$

Analytical Laplace inversions is not applicable for back transformation of the solutions in the actual time domain $\tau$. Thus, the numerical Laplace inversion is applied for back transformation. ${ }^{57,59}$

\section{Type 2: Danckwerts BCs}

The Laplace transformations of Danckwerts BCs given by Eqs. (21a) and (21b) are given as

$$
\bar{c}_{b_{i}}(s, 0)=\frac{c_{b_{i}, \text { inj }}}{s}\left(1-e^{-s \tau_{\mathrm{inj}}}\right)+\left.\frac{1}{P e_{b}} \frac{d \bar{c}_{b_{i}}}{d x}\right|_{x=0}, \quad \frac{d \bar{c}_{b_{i}}}{d x}(s, x=1)=0, \quad i=1,2 .
$$


Following the same solution procedure of Subsection 3.1, the Laplace domain solutions can be obtained as

$$
\begin{aligned}
\bar{c}_{b_{1}}(s, x)= & \frac{c_{b_{1}, \mathrm{inj}}\left(1-e^{-s \tau_{\mathrm{inj}}}\right)}{s} \frac{\left(m_{2}-m_{1}\right) e^{m_{2}+m_{1}}}{\left(1-\frac{m_{1}}{P e_{b}}\right) m_{2} e^{m_{2}}-\left(1-\frac{m_{2}}{P e_{b}}\right) m_{1} e^{m_{1}}}, \\
\bar{c}_{b_{2}}(s, x)= & \frac{-\xi_{p_{2}} A(s)}{\phi_{1}(s)-\phi_{2}(s)} \frac{c_{b_{1}, \mathrm{inj}}\left(1-e^{-s \tau_{\mathrm{inj}}}\right)}{s} \frac{\left(m_{2}-m_{1}\right) e^{m_{2}+m_{1}}}{\left(1-\frac{m_{1}}{P e_{b}}\right) m_{2} e^{m_{2}}-\left(1-\frac{m_{2}}{P e_{b}}\right) m_{1} e^{m_{1}}} \\
& +\frac{\left(1-e^{-s \tau_{\mathrm{inj}}}\right)}{s} \frac{\left[c_{\mathrm{b}_{2}, \mathrm{inj}}-\left(\frac{-\xi_{p_{2}} F A(s)}{\phi_{1}(s)-\phi_{2}(s)}\right) c_{b_{1}, \mathrm{inj}}\right]\left(m_{3}-m_{4}\right) e^{\left(m_{4}+m_{3}\right)}}{\left(1-\frac{m_{4}}{P e_{b}}\right) m_{3} e^{m_{3}}-\left(1-\frac{m_{3}}{P e_{b}}\right) m_{4} e^{m_{4}}} .
\end{aligned}
$$

There is no possibility to apply analytical back transformation on these Laplace domain solutions. However, the numerical Laplace inversion can be applied to obtain a discrete solution in time. In this technique, the integral of inverse Laplace transformation is approximated by Fourier series. ${ }^{57,59}$

\section{GRM for core-shell particles considering reversible Re-}

\section{action $A \rightleftharpoons B$}

This section presents a linear general rate model for simulating reversible reactions in a chromatographic reactor packed with core-shell particles. In this case, the reactant $A$ (component 1 ) is injected to the column which converts to product $B$ (component 2 ) with a reaction rate constant $\nu_{1}$. Due to reversible reaction, product $B$ also partly converts back into the reactant $A$ having a reaction rate constant $\nu_{2}$. In the current setup, the mass balances in the bulk of liquid are expressed as

$$
\frac{\partial c_{b_{i}}}{\partial t}+u \frac{\partial c_{b_{i}}}{\partial z}=D_{b} \frac{\partial^{2} c_{b_{i}}}{\partial z^{2}}-\frac{3}{R_{p}} F_{b} k_{\mathrm{ext}_{i}}\left(c_{b_{i}}-\left.c_{p_{i}}\right|_{r=R_{p}}\right), \quad i=1,2 .
$$


For dynamics inside the particle, the model equations are formulated as

$$
\begin{aligned}
\epsilon_{p} \frac{\partial c_{p_{i}}}{\partial t} & +\left(1-\epsilon_{p}\right) \frac{\partial q_{p_{i}}^{*}}{\partial t}=\frac{1}{r^{2}} \frac{\partial}{\partial r}\left[r^{2}\left(\epsilon_{p} D_{p_{i}} \frac{\partial c_{p_{i}}}{\partial r}+\left(1-\epsilon_{p}\right) D_{s_{i}} \frac{\partial q_{p_{i}}^{*}}{\partial r}\right)\right] \\
& \mp\left(1-\epsilon_{p}\right)\left(\nu_{1} a_{1} c_{p_{1}}-\nu_{2} a_{2} c_{p_{2}}\right), \quad i=1,2 .
\end{aligned}
$$

where $q_{p_{i}}^{*}(i=1,2)$ are given by Eq. (4). After using the dimensionless variables in Eq. (8), the isotherms $q_{p_{i}}^{*}$ in Eq. (4) and substitution in Eq. (13), the above equations can be rewritten as

$$
\begin{aligned}
& \frac{\partial c_{b_{i}}}{\partial \tau}+\frac{\partial c_{b_{i}}}{\partial x}=\frac{1}{P e_{b}} \frac{\partial^{2} c_{b_{i}}}{\partial x^{2}}-\xi_{p_{i}}\left(c_{b_{i}}-\left.c_{p_{i}}\right|_{\rho=1}\right) \\
& (1-\beta)^{2} a_{i}^{*} \frac{\partial}{\partial \tau}\left[(\gamma(1-\beta)+\beta) c_{p_{i}}\right]-\eta_{b_{i}} \frac{\partial^{2}}{\partial \gamma^{2}}\left[(\gamma(1-\beta)+\beta) c_{p_{i}}\right] \pm\left(1-\epsilon_{p}\right)\left((1-\beta)^{2} \omega_{1} a_{1}\right. \\
& \left.\left[(\gamma(1-\beta)+\beta) c_{p_{1}}\right]-(1-\beta)^{2} \omega_{2} a_{2}\left[(\gamma(1-\beta)+\beta) c_{p_{2}}\right]\right), \quad i=1,2
\end{aligned}
$$

The same initial and boundary conditions are used as given by Eqs. (18)-(19). The application of Laplace transformation on the above equations yields

$$
\begin{aligned}
& s \bar{c}_{b_{i}}+\frac{d \bar{c}_{b_{i}}}{d \gamma}=\frac{1}{P e_{b}} \frac{d^{2} \bar{c}_{b_{i}}}{d \gamma^{2}}-\xi_{p_{i}}\left(\bar{c}_{b_{i}}-\left.\bar{c}_{p_{i}}\right|_{\gamma=1}\right) \\
& (1-\beta)^{2} a_{i}^{*}\left[(\gamma(1-\beta)+\beta) \bar{c}_{p_{i}}\right] s-\eta_{p_{i}} \frac{d^{2}}{d \gamma^{2}}\left[(\gamma(1-\beta)+\beta) \bar{c}_{p_{i}}\right] \\
& \pm\left(1-\epsilon_{p}\right)\left((1-\beta)^{2} \omega_{1} a_{1}\left[(\gamma(1-\beta)+\beta) \bar{c}_{p_{1}}\right]-(1-\beta)^{2} \omega_{2} a_{2}\left[(\gamma(1-\beta)+\beta) \bar{c}_{p_{2}}\right]\right)=0,
\end{aligned}
$$

In matrix natation, Eqs. (63) can be expressed as

$$
\frac{d^{2}}{d \gamma^{2}}\left(\begin{array}{c}
(\gamma(1-\beta)+\beta) \bar{c}_{p_{1}} \\
(\gamma(1-\beta)+\beta) \bar{c}_{p_{2}}
\end{array}\right)-(1-\beta)^{2}\left[\begin{array}{cc}
\frac{a_{1}^{*} s+\omega_{1} \epsilon_{p}}{\eta_{p_{1}}} & -\frac{\omega_{2} a_{2}\left(1-\epsilon_{p}\right)}{\eta_{p_{1}}} \\
-\frac{\omega_{1} a_{1}\left(1-\epsilon_{p}\right)}{\eta_{p_{2}}} & \frac{a_{2}^{*} s+\omega_{2} a_{2}\left(1-\epsilon_{p}\right)}{\eta_{p_{2}}}
\end{array}\right]\left(\begin{array}{c}
(\gamma(1-\beta)+\beta) \bar{c}_{p_{1}} \\
(\gamma(1-\beta)+\beta) \bar{c}_{p_{2}}
\end{array}\right)=\left(\begin{array}{l}
0 \\
0
\end{array}\right) .
$$


The reaction coefficient matrix $\left[B^{\prime}\right]$ of Eq. (64) is given as

$$
B^{\prime}=\left[\begin{array}{cc}
\frac{a_{1}^{*} s+\omega_{1} \epsilon_{p}}{\eta_{p_{1}}} & -\frac{\omega_{2} a_{2}\left(1-\epsilon_{p}\right)}{\eta_{p_{1}}} \\
-\frac{\omega_{1} a_{1}\left(1-\epsilon_{p}\right)}{\eta_{p_{2}}} & \frac{a_{2}^{*} s+\omega_{2} a_{2}\left(1-\epsilon_{p}\right)}{\eta_{p_{2}}}
\end{array}\right]
$$

The eigenvalues and eigenvectors of the matrix $\left[B^{\prime}\right]$ are given as:

$$
\lambda_{1,2}^{\prime}=\frac{\beta_{1} \pm \sqrt{\beta_{1}^{2}-4 \beta_{2}}}{2}, \quad x_{1,2}^{\prime}=\left(\begin{array}{c}
\lambda_{1,2}^{\prime}-\frac{a_{2}^{*} s+\omega_{2} a_{2}\left(1-\epsilon_{p}\right)}{\eta_{p_{2}}} \\
-\frac{\omega_{1} a_{1}\left(1-\epsilon_{p}\right)}{\eta_{p_{2}}}
\end{array}\right)
$$

where

$\beta_{1}(s)=\frac{a_{1}^{*} s}{\eta_{p_{1}}}+\frac{a_{2}^{*} s}{\eta_{p_{2}}}+\frac{\left(1-\epsilon_{p}\right) \omega_{1} a_{1}}{\eta_{p_{1}}}+\frac{\left(1-\epsilon_{p}\right) \omega_{2} a_{2}}{\eta_{p_{2}}}, \quad \beta_{2}(s)=\frac{a_{1}^{*} a_{2}^{*} s^{2}}{\eta_{p_{1}} \eta_{p_{2}}}+\frac{a_{2}^{*} s\left(1-\epsilon_{p}\right) \omega_{1} a_{1}}{\eta_{p_{1}} \eta_{p_{2}}}+\frac{a_{1}^{*} s\left(1-\epsilon_{p}\right) \omega_{2} a_{2}}{\eta_{p_{1}} \eta_{p_{2}}}$.

Thus, we have the following linear transformation

$$
\left(\begin{array}{c}
\bar{c}_{p_{1}} \\
\bar{c}_{p_{2}}
\end{array}\right)=\left[\begin{array}{cc}
\lambda_{1}^{\prime}-\frac{a_{2}^{*} s+\omega_{2} a_{2}\left(1-\epsilon_{p}\right)}{\eta_{p_{2}}} & \lambda_{2}^{\prime}-\frac{a_{2}^{*} s+\omega_{2} a_{2}\left(1-\epsilon_{p}\right)}{\eta_{p_{2}}} \\
-\frac{\omega_{1} a_{1}\left(1-\epsilon_{p}\right)}{\eta_{p_{2}}} & -\frac{\omega_{1} a_{1}\left(1-\epsilon_{p}\right)}{\eta_{p_{2}}}
\end{array}\right]\left(\begin{array}{l}
\bar{b}_{p_{1}} \\
\bar{b}_{p_{2}}
\end{array}\right)
$$

Applying the above linear transformation on Eq. (64), we get

$$
\frac{d^{2}}{d \gamma^{2}}\left(\begin{array}{c}
\gamma(1-\beta)+\beta) \bar{b}_{p_{1}} \\
\gamma(1-\beta)+\beta) \bar{b}_{p_{2}}
\end{array}\right)-(1-\beta)^{2}\left[\begin{array}{cc}
\lambda_{1}^{\prime} & 0 \\
0 & \lambda_{2}^{\prime}
\end{array}\right]\left(\begin{array}{l}
\gamma(1-\beta)+\beta) \bar{b}_{p_{1}} \\
\gamma(1-\beta)+\beta) \bar{b}_{p_{2}}
\end{array}\right)=\left(\begin{array}{l}
0 \\
0
\end{array}\right)
$$

Eq. (69) represents a system of two independent ODEs. Their explicit solutions are given as

$$
\bar{b}_{p_{1}}(s, x, \gamma)=\frac{1}{\gamma(1-\beta)+\beta}\left[A_{1}^{\prime} e^{(1-\beta) \sqrt{\lambda_{1}^{\prime}} \gamma}+B_{1}^{\prime} e^{-(1-\beta) \sqrt{\lambda_{1}^{\prime}} \gamma}\right]
$$




$$
\bar{b}_{p_{2}}(s, x, \gamma)=\frac{1}{\gamma(1-\beta)+\beta}\left[A_{2}^{\prime} e^{(1-\beta) \sqrt{\lambda_{2}^{\prime}} \gamma}+B_{2}^{\prime} e^{-(1-\beta) \sqrt{\lambda_{2}^{\prime}} \gamma}\right]
$$

From Eqs. (19) and (68), one can easily find

$$
\left.\frac{\partial b_{p_{i}}(s, \gamma)}{\partial \gamma}\right|_{\gamma=0}=0, \quad i=1,2
$$

On using this boundary conditions in Eq. (70), we get $A_{1}^{\prime}=\frac{\left(\beta \sqrt{\lambda_{1}^{\prime}}+1\right)}{\left(\beta \sqrt{\lambda_{1}^{\prime}}-1\right)} B_{1}^{\prime}$ and $A_{2}^{\prime}=$ $\frac{\left(\beta \sqrt{\lambda_{2}^{\prime}}+1\right)}{\left(\beta \sqrt{\lambda_{2}^{\prime}}-1\right)} B_{2}^{\prime}$. Thus, Eq. (70) reduces to

$$
\begin{aligned}
& b_{p_{1}}(s, x, \gamma)=\frac{2 A_{1}^{\prime}}{\gamma(1-\beta)+\beta)} \beta \sqrt{\lambda_{1}^{\prime}} \cosh \left((1-\beta) \sqrt{\lambda_{1}^{\prime}} \gamma\right)+\frac{2 A_{1}^{\prime}}{\gamma(1-\beta)+\beta)} \sinh \left((1-\beta) \sqrt{\lambda_{1}^{\prime}} \gamma\right), \\
& b_{p_{2}}(s, x, \gamma)=\frac{2 A_{2}^{\prime}}{\gamma(1-\beta)+\beta)} \beta \sqrt{\lambda_{2}^{\prime}} \cosh \left((1-\beta) \sqrt{\lambda_{2}^{\prime}} \gamma\right)+\frac{2 A_{2}^{\prime}}{\gamma(1-\beta)+\beta)} \sinh \left((1-\beta) \sqrt{\lambda_{2}^{\prime}} \gamma\right) .
\end{aligned}
$$

By using the transformation in Eq. (68) and values of $b_{i}$ in Eq. (72), we obtain

$$
\begin{aligned}
c_{p_{1}}(s, x, \gamma)= & \left.\lambda_{1}^{\prime}-\frac{a_{2}^{*} s+\omega_{2} a_{2}\left(1-\epsilon_{p}\right)}{\eta_{p_{2}}}\right] \frac{2 A_{1}^{\prime}}{\gamma(1-\beta)+\beta)}\left[\beta \sqrt{\lambda_{1}^{\prime}} \cosh \left((1-\beta) \sqrt{\lambda_{1}^{\prime}} \gamma\right)\right] \\
& +\sinh \left(\sqrt{\lambda_{1}^{\prime}} \gamma\right)+\sinh \left((1-\beta) \sqrt{\lambda_{1}^{\prime}} \gamma\right) \\
& \left.+\left[\lambda_{2}^{\prime}-\frac{a_{2}^{*} s+\omega_{2} a_{2}\left(1-\epsilon_{p}\right)}{\eta_{p_{2}}}\right] \frac{2 A_{2}^{\prime}}{\gamma} \gamma(1-\beta)+\beta\right)\left[\beta \sqrt{\lambda_{2}^{\prime}} \cosh \left((1-\beta) \sqrt{\lambda_{2}^{\prime}} \gamma\right)\right]+\sinh \left(\sqrt{\lambda_{2}^{\prime}} \gamma\right) \\
& +\sinh \left((1-\beta) \sqrt{\lambda_{2}^{\prime}} \gamma\right) \\
c_{p_{2}}(s, x, \gamma)= & -\frac{\omega_{1} a_{1}\left(1-\epsilon_{p}\right)}{\eta_{p_{2}}}\left[\frac{2 A_{1}^{\prime}}{\gamma(1-\beta)+\beta)}\left[\beta \sqrt{\lambda_{1}^{\prime}} \cosh \left((1-\beta) \sqrt{\lambda_{1}^{\prime}} \gamma\right)\right]+\sinh \left(\sqrt{\lambda_{1}^{\prime}} \gamma\right)\right. \\
& \left.+\sinh \left((1-\beta) \sqrt{\lambda_{1}^{\prime}} \gamma\right)+\frac{2 A_{2}^{\prime}}{\gamma} \gamma(1-\beta)+\beta\right)\left[\beta \sqrt{\lambda_{2}^{\prime}} \cosh \left((1-\beta) \sqrt{\lambda_{2}^{\prime}} \gamma\right)\right]+\sinh \left(\sqrt{\lambda_{2}^{\prime}} \gamma\right) \\
& \left.+\sinh \left((1-\beta) \sqrt{\lambda_{2}^{\prime}} \gamma\right)\right]
\end{aligned}
$$

By using second boundary condition at $\rho=1$ (c.f. Eq. (19)) in Eqs. (74) and (75), we get 
a system of two equations in term of $A_{1}^{\prime}$ and $A_{2}^{\prime}$. On solving those equations, we obtain

$$
\begin{aligned}
& A_{1}^{\prime}=\frac{1}{2 \sinh \left((1-\beta) \sqrt{\lambda_{1}^{\prime}}\right)}\left[\frac{\alpha_{1}(s)}{A(s)} \bar{c}_{b_{1}}+\frac{\alpha_{2}(s)}{A(s)} \frac{\left.\eta_{p_{2}}\left(\lambda_{2}^{\prime}-\frac{a_{2}^{*} s+\omega_{2} a_{2}\left(1-\epsilon_{p}\right)}{\eta_{p_{2}}}\right)\right)}{\left(1-\epsilon_{p}\right) a_{1} \omega_{1}} \bar{c}_{b_{2}}\right], \\
& A_{2}^{\prime}=-\frac{1}{2 \sinh \left((1-\beta) \sqrt{\lambda_{2}^{\prime}}\right)}\left[\frac{\alpha_{3}(s)}{A(s)} \bar{c}_{b_{1}}+\frac{\alpha_{4}(s)}{A(s)} \frac{\left.\eta_{p_{2}}\left(\lambda_{1}^{\prime}-\frac{a_{2}^{*} s+\omega_{2} a_{2}\left(1-\epsilon_{p}\right)}{\eta_{p_{2}}}\right)\right)}{\left(1-\epsilon_{p}\right) a_{1} \omega_{1}} \bar{c}_{b_{2}}\right],
\end{aligned}
$$

where

$$
\begin{aligned}
\alpha_{1}(s)= & B i_{p_{1}}\left(\left[\beta \sqrt{\lambda_{2}^{\prime}} \operatorname{coth}\left((1-\beta) \sqrt{\lambda_{2}^{\prime}} \gamma\right)+1\right]\left(B i_{p_{2}}-1\right)+\left[\beta \lambda_{2}^{\prime}+\sqrt{\lambda_{2}^{\prime}} \operatorname{coth}\left((1-\beta) \sqrt{\lambda_{2}^{\prime}}\right)\right]\right) \\
\alpha_{2}(s)= & B i_{p_{2}}\left(\left[\beta \sqrt{\lambda_{2}^{\prime}} \operatorname{coth}\left((1-\beta) \sqrt{\lambda_{2}^{\prime}} \gamma\right)+1\right]\left(B i_{p_{1}}-1\right)+\left[\beta \lambda_{2}^{\prime}+\sqrt{\lambda_{2}^{\prime}} \operatorname{coth}\left((1-\beta) \sqrt{\lambda_{2}^{\prime}}\right)\right]\right) \\
\alpha_{3}(s)= & B i_{p_{1}}\left(\left[\beta \sqrt{\lambda_{1}^{\prime}} \operatorname{coth}\left((1-\beta) \sqrt{\lambda_{1}^{\prime}} \gamma\right)+1\right]\left(B i_{p_{2}}-1\right)+\left[\beta \lambda_{1}^{\prime}+\sqrt{\lambda_{1}^{\prime}} \operatorname{coth}\left((1-\beta) \sqrt{\lambda_{1}^{\prime}}\right)\right]\right) \\
\alpha_{4}(s)= & B i_{p_{2}}\left(\left[\beta \sqrt{\lambda_{1}^{\prime}} \operatorname{coth}\left((1-\beta) \sqrt{\lambda_{1}^{\prime}} \gamma\right)+1\right]\left(B i_{p_{1}}-1\right)+\left[\beta \lambda_{1}^{\prime}+\sqrt{\lambda_{1}^{\prime}} \operatorname{coth}\left((1-\beta) \sqrt{\lambda_{1}^{\prime}}\right)\right]\right) \\
A(s)= & {\left[\lambda_{1}^{\prime}-\frac{a_{2}^{*} s+\omega_{2} a_{2}\left(1-\epsilon_{p}\right)}{\eta_{p_{2}}}\right]\left[\beta \sqrt{\lambda_{1}^{\prime}} \operatorname{coth}\left((1-\beta) \sqrt{\lambda_{1}^{\prime}} \gamma\right)+1\right]\left(B i_{p_{1}}-1\right)+\left[\beta \lambda_{1}^{\prime}+\sqrt{\lambda_{1}^{\prime}} \operatorname{coth}\left(\sqrt{\lambda_{1}^{\prime}}\right)\right] } \\
& {\left[\beta \sqrt{\lambda_{2}^{\prime}} \operatorname{coth}\left((1-\beta) \sqrt{\lambda_{2}^{\prime}} \gamma\right)+1\right]\left(B i_{p_{2}}-1\right)+\left[\beta \lambda_{2}^{\prime}+\sqrt{\lambda_{2}^{\prime}} \operatorname{coth}\left(\sqrt{\lambda_{2}^{\prime}}\right)\right]-\left(\lambda_{2}^{\prime}-\frac{a_{2}^{*} s+\omega_{2} a_{2}\left(1-\epsilon_{p}\right)}{\eta_{p_{2}}}\right) } \\
& {\left[\beta \sqrt{\lambda_{2}^{\prime}} \operatorname{coth}\left((1-\beta) \sqrt{\lambda_{2}^{\prime}} \gamma\right)+1\right]\left(B i_{p_{1}}-1\right)+\left[\beta \lambda_{2}^{\prime}+\sqrt{\lambda_{2}^{\prime}} \operatorname{coth}\left(\sqrt{\lambda_{2}^{\prime}}\right)\right]\left[\beta \sqrt { \lambda _ { 1 } ^ { \prime } } \operatorname { c o t h } \left((1-\beta) \sqrt{\left.\left.\lambda_{1}^{\prime} \gamma\right)+1\right]}\right.\right.} \\
& +\left(B i_{p_{2}}-1\right)\left[\beta \lambda_{1}^{\prime}+\sqrt{\lambda_{1}^{\prime}} \operatorname{coth}\left(\sqrt{\lambda_{1}^{\prime}}\right)\right] .
\end{aligned}
$$

Thus, Eqs. (74) and (75) take the forms

$$
\begin{aligned}
\left.c_{p_{1}}\right|_{\gamma=1}= & {\left[\frac{\left[\beta \sqrt{\lambda_{1}^{\prime}} \operatorname{coth}\left((1-\beta) \sqrt{\lambda_{1}^{\prime}} \gamma\right)+1\right] \alpha_{1}(s)}{A(s)}\left(\lambda_{1}^{\prime}-\frac{a_{2}^{*} s+\omega_{2} a_{2}\left(1-\epsilon_{p}\right)}{\eta_{p_{2}}}\right)\right.} \\
& \left.-\frac{\left[\beta \sqrt{\lambda_{2}^{\prime}} \operatorname{coth}\left((1-\beta) \sqrt{\lambda_{2}^{\prime}} \gamma\right)+1\right] \alpha_{3}(s)}{A(s)}\left(\lambda_{2}^{\prime}-\frac{a_{2}^{*} s+\omega_{2} a_{2}\left(1-\epsilon_{p}\right)}{\eta_{p_{2}}}\right)\right] \bar{c}_{b_{1}}
\end{aligned}
$$




$$
\begin{aligned}
& -\frac{\epsilon_{p} \omega_{2}}{\eta_{p_{1}}}\left[\beta \sqrt{\lambda_{2}^{\prime}} \operatorname{coth}\left((1-\beta) \sqrt{\lambda_{2}^{\prime}} \gamma\right)+1\right]\left[\frac{\alpha_{2}(s)}{A(s)}-\frac{\alpha_{4}(s)}{A(s)}\right] \bar{c}_{b_{2}} \\
\left.c_{p_{2}}\right|_{\gamma=1}= & -\frac{\epsilon_{p} \omega_{1}}{\eta_{p_{2}}}\left[\frac{\left[\beta \sqrt{\lambda_{1}^{\prime}} \operatorname{coth}\left((1-\beta) \sqrt{\lambda_{1}^{\prime}} \gamma\right)+1\right] \alpha_{1}(s)}{A(s)}-\frac{\left[\beta \sqrt{\lambda_{2}^{\prime}} \operatorname{coth}\left((1-\beta) \sqrt{\lambda_{2}^{\prime}} \gamma\right)+1\right] \alpha_{3}(s)}{A(s)}\right] \bar{c}_{b_{1}} \\
& +\left[\frac{\left[\beta \sqrt{\lambda_{2}^{\prime}} \operatorname{coth}\left((1-\beta) \sqrt{\lambda_{2}^{\prime}} \gamma\right)+1\right] \alpha_{4}(s)}{A(s)}\left(\lambda_{1}^{\prime}-\frac{a_{2}^{*} s+\omega_{2} a_{2}\left(1-\epsilon_{p}\right)}{\eta_{p_{2}}}\right)\right. \\
& \left.-\frac{\left[\beta \sqrt{\lambda_{1}^{\prime}} \operatorname{coth}\left((1-\beta) \sqrt{\lambda_{1}^{\prime}} \gamma\right)+1\right] \alpha_{2}(s)}{A(s)}\left(\lambda_{2}^{\prime}-\frac{a_{2}^{*} s+\omega_{2} a_{2}\left(1-\epsilon_{p}\right)}{\eta_{p_{2}}}\right)\right] \bar{c}_{b_{2}} .
\end{aligned}
$$

By using Eqs. (83) and (84) in Eqs. (62), we get the following system of ODEs

$$
\frac{d^{2}}{d x^{2}}\left(\begin{array}{c}
\bar{c}_{b_{1}} \\
\bar{c}_{b_{2}}
\end{array}\right)-P e_{b} \frac{d}{d x}\left(\begin{array}{c}
\bar{c}_{b_{1}} \\
\bar{c}_{b_{2}}
\end{array}\right)+\left[\begin{array}{cc}
-r_{1}-s P e_{b} & r_{2} \\
r_{3} & -r_{4}-s P e_{b}
\end{array}\right]\left(\begin{array}{l}
\bar{c}_{b_{1}} \\
\bar{c}_{b_{2}}
\end{array}\right)=\left(\begin{array}{l}
0 \\
0
\end{array}\right),
$$

where

$$
\begin{aligned}
r_{1}= & P e_{b} \xi_{p_{1}}\left[1-\frac{\left[\beta \sqrt{\lambda_{1}^{\prime}} \operatorname{coth}\left((1-\beta) \sqrt{\lambda_{1}^{\prime}} \gamma\right)+1\right] \alpha_{1}(s)}{A(s)}\left(\lambda_{1}^{\prime}-\frac{a_{2}^{*} s+\omega_{2} a_{2}\left(1-\epsilon_{p}\right)}{\eta_{p_{2}}}\right)\right. \\
& \left.+\frac{\left[\beta \sqrt{\lambda_{2}^{\prime}} \operatorname{coth}\left((1-\beta) \sqrt{\lambda_{2}^{\prime}} \gamma\right)+1\right] \alpha_{3}(s)}{A(s)}\left(\lambda_{2}^{\prime}-\frac{a_{2}^{*} s+\omega_{2} a_{2}\left(1-\epsilon_{p}\right)}{\eta_{p_{2}}}\right)\right], \\
r_{2}= & -P e_{b} \xi_{p_{1}} \frac{\left(1-\epsilon_{p}\right) a_{2} \omega_{2}}{\eta_{p_{1}}}\left[\frac{\left[\beta \sqrt{\lambda_{2}^{\prime}} \operatorname{coth}\left((1-\beta) \sqrt{\lambda_{2}^{\prime}} \gamma\right)+1\right] \alpha_{2}(s)}{A(s)}-\frac{\left.\left[\beta \sqrt{\lambda_{1}^{\prime}} \operatorname{coth}\left((1-\beta) \sqrt{\lambda_{1}^{\prime}} \gamma\right)+1\right] \alpha_{4}(s)\right]}{A(s)}\right] \\
r_{3}= & -P e_{b} \xi_{p_{2}} \frac{\left(1-\epsilon_{p}\right) a_{1} \omega_{1}}{\eta_{p_{2}}}\left[\frac{\left[\beta \sqrt{\lambda_{1}^{\prime}} \operatorname{coth}\left((1-\beta) \sqrt{\lambda_{1}^{\prime}} \gamma\right)+1\right] \alpha_{1}(s)}{A(s)}-\frac{\left[\beta \sqrt{\lambda_{2}^{\prime}} \operatorname{coth}\left((1-\beta) \sqrt{\lambda_{2}^{\prime}} \gamma\right)+1\right] \alpha_{3}(s)}{A(s)}\right] \\
r_{4}= & P e_{b} \xi_{p_{2}}\left[1-\frac{\left[\beta \sqrt{\lambda_{2}^{\prime}} \operatorname{coth}\left((1-\beta) \sqrt{\lambda_{2}^{\prime}} \gamma\right)+1\right] \alpha_{4}(s)}{A(s)}\left(\lambda_{1}^{\prime}-\frac{a_{2}^{*} s+\omega_{2} a_{2}\left(1-\epsilon_{p}\right)}{\eta_{p_{2}}}\right)\right. \\
& \left.+\frac{\left[\beta \sqrt{\lambda_{1}^{\prime}} \operatorname{coth}\left((1-\beta) \sqrt{\lambda_{1}^{\prime}} \gamma\right)+1\right] \alpha_{2}(s)}{A(s)}\left(\lambda_{2}^{\prime}-\frac{a_{2}^{*} s+\omega_{2} a_{2}\left(1-\epsilon_{p}\right)}{\eta_{p_{2}}}\right)\right]
\end{aligned}
$$

By following the solution procedure of Section 3, we get the following Laplace domain solutions of the system in Eq. (85) for the considered two sets of BCs. 


\section{Type 1: Dirichlet BCs}

Here, the boundary conditions in Eqs. (20a) and (20b) are taken into account. The Laplace domain solutions are given as

$$
\begin{aligned}
\bar{c}_{b_{1}}(s, x)= & \frac{\left(1-e^{-s \tau_{\mathrm{inj}}}\right) \lambda_{3}^{\prime \prime}\left(r_{3} c_{b_{1}, \mathrm{inj}}-\lambda_{4}^{\prime \prime} c_{b_{2}, \mathrm{inj}}\right)\left(m_{2}^{\prime} e^{m_{2}^{\prime}+m_{1}^{\prime} x}-m_{1}^{\prime} e^{m_{1}^{\prime}+m_{2}^{\prime} x}\right)}{s r_{3}\left(\lambda_{3}^{\prime \prime}-\lambda_{4}^{\prime \prime}\right)\left(m_{2}^{\prime} e^{m_{2}^{\prime}}-m_{1}^{\prime} e^{m_{1}^{\prime}}\right)} \\
& -\frac{\left(1-e^{-s \tau_{\mathrm{inj}}}\right) \lambda_{4}^{\prime \prime}\left(r_{3} c_{b_{1}, \mathrm{inj}}-\lambda_{3}^{\prime \prime} c_{b_{2}, \mathrm{inj}}\right)\left(m_{4}^{\prime} e^{m_{4}^{\prime}+m_{3}^{\prime} x}-m_{3}^{\prime} e^{m_{3}^{\prime}+m_{4}^{\prime} x}\right)}{s r_{3}\left(\lambda_{3}^{\prime \prime}-\lambda_{4}^{\prime \prime}\right)\left(m_{4}^{\prime} e^{m_{4}^{\prime}}-m_{3}^{\prime} e^{m_{3}^{\prime}}\right)} \\
\bar{c}_{b_{2}}(s, x)= & \frac{\left(1-e^{-s \tau_{\mathrm{inj}}}\right)\left(r_{3} c_{b_{1}, \mathrm{inj}}-\lambda_{4}^{\prime \prime} c_{b_{2}, \mathrm{inj}}\right)\left(m_{2}^{\prime} e^{m_{2}^{\prime}+m_{1}^{\prime} x}-m_{1}^{\prime} e^{m_{1}^{\prime}+m_{2}^{\prime} x}\right)}{s\left(\lambda_{3}^{\prime \prime}-\lambda_{4}^{\prime \prime}\right)\left(m_{2}^{\prime} e^{m_{2}^{\prime}}-m_{1}^{\prime} e^{m_{1}^{\prime}}\right)} \\
& -\frac{\left(1-e^{-s \tau_{\mathrm{inj}}}\right)\left(r_{3} c_{b_{1}, \mathrm{inj}}-\lambda_{3}^{\prime \prime} c_{b_{2}, \mathrm{inj}}\right)\left(m_{4}^{\prime} e^{m_{4}^{\prime}+m_{3}^{\prime} x}-m_{3}^{\prime} e^{m_{3}^{\prime}+m_{4}^{\prime} x}\right)}{s\left(\lambda_{3}^{\prime \prime}-\lambda_{4}^{\prime \prime}\right)\left(m_{4}^{\prime} e^{m_{4}^{\prime}}-m_{3}^{\prime} e^{m_{3}^{\prime}}\right)}
\end{aligned}
$$

where

$$
\begin{aligned}
\lambda_{1,2}^{\prime \prime} & =-\frac{1}{2}\left[r_{1}+r_{4}+2 s P e_{b} \mp \sqrt{4\left(r_{2} r_{3}-r_{1} r_{4}\right)+\left(r_{1}+r_{4}\right)^{2}}\right], \\
\lambda_{3,4}^{\prime \prime} & =-\frac{1}{2}\left[r_{1}-r_{4} \mp \sqrt{4\left(r_{2} r_{3}-r_{1} r_{4}\right)+\left(r_{1}+r_{4}\right)^{2}}\right] . \\
m_{1,2}^{\prime} & =\frac{P e_{b} \pm \sqrt{P e_{b}^{2}-4 \lambda_{1}^{\prime \prime}}}{2}, \quad m_{3,4}^{\prime}=\frac{P e_{b} \pm \sqrt{P e_{b}^{2}-4 \lambda_{2}^{\prime \prime}}}{2} .
\end{aligned}
$$

Once again, the analytical Laplace inversion is not applicable. For that reason, the numerical Laplace inversion of Durbin is applied to get back solutions in the actual time domain. ${ }^{59}$

\section{Type 2: Danckwerts BCs}

Here, the BCs in Eq. (21a) and (21b) are applied. The Laplace domain are expressed as

$$
\begin{aligned}
\bar{c}_{b_{1}}(s, x) & =\frac{\left(1-e^{-s \tau_{\mathrm{inj}}}\right) \lambda_{3}^{\prime \prime}\left(r_{3} c_{b_{1}, \mathrm{inj}}-\lambda_{4}^{\prime \prime} c_{b_{2}, \mathrm{inj}}\right)\left(m_{1}^{\prime} e^{m_{1}^{\prime}+m_{2}^{\prime} x}-m_{2}^{\prime} e^{m_{2}^{\prime}+m_{1}^{\prime} x}\right)}{r_{3} s\left(\lambda_{3}^{\prime \prime}-\lambda_{4}^{\prime \prime}\right)\left[\left(1-\frac{m_{2}^{\prime}}{P e_{b}}\right) m_{1}^{\prime} e^{m_{1}^{\prime}}-\left(1-\frac{m_{1}^{\prime}}{P e_{b}}\right) m_{2}^{\prime} e^{m_{2}^{\prime}}\right]} \\
& -\frac{\left(1-e^{-s \tau_{\mathrm{inj}}}\right) \lambda_{4}^{\prime \prime}\left(r_{3} c_{b_{1}, \mathrm{inj}}-\lambda_{3}^{\prime \prime} c_{b_{2}, \mathrm{inj}}\right)\left(m_{3}^{\prime} e^{m_{3}^{\prime}+m_{4}^{\prime} x}-m_{4}^{\prime} e^{m_{4}^{\prime}+m_{3}^{\prime} x}\right)}{r_{3} s\left(\lambda_{3}^{\prime \prime}-\lambda_{4}^{\prime \prime}\right)\left[\left(1-\frac{m_{4}^{\prime}}{P e_{b}}\right) m_{3}^{\prime} e^{m_{3}^{\prime}}-\left(1-\frac{m_{3}^{\prime}}{P e_{b}}\right) m_{4}^{\prime} e^{m_{4}^{\prime}}\right]}
\end{aligned}
$$




$$
\begin{aligned}
\bar{c}_{b_{2}}(s, x) & =\frac{\left(1-e^{-s \tau_{\mathrm{inj}}}\right)\left(r_{3} c_{b_{1}, \mathrm{inj}}-\lambda_{4}^{\prime \prime} c_{b_{2}, \mathrm{inj}}\right)\left(m_{1}^{\prime} e^{m_{1}^{\prime}+m_{2}^{\prime} x}-m_{2}^{\prime} e^{m_{2}^{\prime}+m_{1}^{\prime} x}\right)}{s\left(\lambda_{3}^{\prime \prime}-\lambda_{4}^{\prime \prime}\right)\left[\left(1-\frac{m_{2}^{\prime}}{P e_{b}}\right) m_{1}^{\prime} e^{m_{1}^{\prime}}-\left(1-\frac{m_{1}^{\prime}}{P e_{b}}\right) m_{2}^{\prime} e^{m_{2}^{\prime}}\right]} \\
& -\frac{\left(1-e^{-s \tau_{\mathrm{inj}}}\right)\left(r_{3} c_{b_{1}, \mathrm{inj}}-\lambda_{3}^{\prime \prime} c_{b_{2}, \mathrm{inj}}\right)\left(m_{3}^{\prime} e^{m_{3}^{\prime}+m_{4}^{\prime} x}-m_{4}^{\prime} e^{m_{4}^{\prime}+m_{3}^{\prime} x}\right)}{s\left(\lambda_{3}^{\prime \prime}-\lambda_{4}^{\prime \prime}\right)\left[\left(1-\frac{m_{4}^{\prime}}{P e_{b}}\right) m_{3}^{\prime} e^{m_{3}^{\prime}}-\left(1-\frac{m_{3}^{\prime}}{P e_{b}}\right) m_{4}^{\prime} e^{m_{4}^{\prime}}\right]} .
\end{aligned}
$$

Here, $\lambda_{i}^{\prime \prime}$ and $m_{i}^{\prime}$ for $i=1,2,3,4$ are given by Eqs. (92)-(94). Once more time, the analytical Laplace inversion is not possible. Therefore, the numerical Laplace inversion of Durbin is applied. ${ }^{59}$

\section{Numerical case studies}

This section presents several numerical case studies to analyze the derived semi-analytical solution. A high resolution finite volume scheme (HR-FVS) is applied to gain further confidence on the correctness of derived semi-analytical solutions. ${ }^{60,61}$ In all test problems, rectangular pulses of the mixture (reactant and product) are injected into the chromatographic reactor for $t_{\mathrm{inj}}=10 \mathrm{~min}$. All the parameters appearing in the model equations are listed in Table 1. These model parameters have been chosen in accordance with ranges typically encountered in HPLC applications.

\section{Problem 1: Linear case of irreversible reaction}

Here, we study the influence of boundary conditions, $\beta, \omega, P e_{b}, B i_{p}$, and $\eta_{p}$, characterizing the core radius fraction, solid phase reaction constant, axial dispersion, film transfer resistance and intraparticle diffusion resistance, on the concentration profiles and numerical moments.

Figure 2 describes the effects of injection when a rectangular pulse of finite width is injected in an empty column $\left(c_{i \text {,init }}=0 \mathrm{~g} / \mathrm{l}\right.$ for $\left.i=1,2\right)$. For the inert core bead value $\beta=0.5$, the concentration profiles of reactant and product are plotted using the Dirichlet BCs. 
In Figure 2(a), the amount of injection for both reactant (component 1) and product ( component 2) is considered the same (i.e. $c_{b_{1} \text {,inj }}=0.5 \mathrm{~g} / \mathrm{l}, c_{b_{2} \text {,inj }}=0.5 \mathrm{~g} / \mathrm{l}$ ), while in Figure $2(\mathrm{~b})$, half amount of component 2 is injected i.e. $c_{b_{2}, \text { inj }}=0.25 \mathrm{~g} / \mathrm{l}$. It can be observed from the plots that the amount of product increases when the same amount of injection is used for both components. As a result more amount of reactant is converted into the product because of the irreversibility of solid phase reaction constant $\omega=0.2$. The semi-analytical and numerical results of HR-FVS are in good agreement with one another in both the cases.

In Figure 3, the effects of core radius fraction, including fully porous beads, are analyzed on the elusion curves of both reactant and product for two different Peclet numbers, i.e. $P e_{b}=73.53$ (Figure 3(a) and Figure 3(b)) and $P e_{b}=7353$ (Figure 3(c) and Figure 3(d)). In Figure 3(a), the behavior of reactant (component 1) is examined, when $\beta$ increases from 0 (fully porous beads) to 0.9 (beads with a very thin shell). As $\beta$ increases, peak of the reactant increases and sharpens due to reduction in intraparticle diffusional mass transfer resistance. In Figure 3(b), the behavior of product (component 2) is explained for the same values of $\beta$. Sharpened and asymmetric peaks of concentration curves are observed for larger values of $\beta$. Efficiency of the column gradually increases for larger value of $\beta$ due to sharpened elusion profiles and column capacity gradually decreases due to smaller residence times of the components. The shorter residence time are due to the loss of binding sites with the larger values of $\beta$, which results in less interaction between the mobile and stationary phases for adsorption and desorption. The concentration profiles in Figure 3(c) and Figure 3(d) have similar behavior but have sharp fronts due to small dispersion coefficient $\left(D_{b}=0.0034\right.$ or $\left.P e_{b}=7353\right)$. In all plots, complete breakthrough curves occur at $\beta=0.9$ because of very low capacity, i.e. feed concentration can be seen at the column outlet. Numerical solutions of HR-FVS are exactly matching with the analytical results for all values of $\beta$ which is an indication for the accuracy of proposed numerical scheme 
and for the correctness of analytical results.

The effects of model parameter $P e_{b}$ is being examined on the concentration profile in Figure 4. It is clear that if axial dispersion is large $\left(P e_{b}=1.25\right)$ elusion profiles for Dirichlet and Danckwert BCs differs from each other. Whereas, for small axial dispersion $\left(P e_{b}=125\right)$, concentration profiles for both boundary conditions overlap each other which quantify the possible back mixing near the column inlet as explained in Figures 4(a) and 4(b). Again, the same effects of $\beta$ on the concentration profiles are observed even for large axial dispersion. The retention times corresponding to the maximum peaks become shorter and peaks are more steeper when $\beta=0.9$. Also, more amount of product is achieved when chromatographic column is packed with fully porous particles $(\beta=0.0)$ as compared to the case when column is packed with core-shell particles $(\beta=0.9)$.

Figure 5 demonstrates how different values of solid phase reaction constant $\omega$ effects the elusion curves for fully porous column $(\beta=0.0)$ and for inert core absorbent column $(\beta=0.9)$. The plots are obtained using Danckwerts boundary conditions and considering $c_{b_{1}, \text { inj }}=0.5 \mathrm{~g} / \mathrm{l}, c_{b_{2}, \text { inj }}=0.5 \mathrm{~g} / \mathrm{l}$, and $c_{i, \text { init }}=0.0 \mathrm{~g} / \mathrm{l}$. It is found that when column is packed with fully porous particles $(\beta=0.0)$, and reaction rate constant $\omega$ increases from 0.0 to 0.6, conversion of reactant into product is faster in both Figures 5(a) and 5(b). While, when the column is packed with core-shell having very thin shell $(\beta=0.9)$, less amount of reactant is converted into product on increasing the value of reaction rate constant $\omega$. In other words, the core-shell particles reduces the amount of product for a given value of the reaction rate constant.

Figure 6(a) describes the effects of Biot number $B i_{p}=\frac{k_{\text {ext }} R_{p}}{D_{\text {effi }}}$ on the reactant (component 1) and Figure 6(b) shows the plots of product (component 2) for three different values of $B i_{p}$. In both figures, it is observed that when the value of $B i_{p}$ increases from 5 to 500 , broadened peaks of the concentration profiles are shifted to steeped peaks. The same effect of $B i_{p}$ is observed for core-shell particles $(\beta=0.9)$. In this case, the residence time inside 
the column reduces again. Also, at $\beta=0.9$, the elution profile of component 2 is more sharped for $B i_{p}=500$ as compared to component 1.

The effects of intraparticle diffusion parameter $\eta_{p}$ on the concentration profiles are discussed in Figures $7(\mathrm{a})$ and $7(\mathrm{~b})$. For small value of $\eta_{p}=0.02$, the diffusion rate is very fast and fluid retention time is shorter for both component 1 and component 2. The tendency is that the breakthrough time for fully porous adsorbents becomes closer to that of inert core adsorbents due to the limitation of the intraparticle diffusion resistance. The zone spreading time means the time from the breakthrough to the saturation of the fixed bed. It can be observed that the zone spreading time for elution curves can be decreased effectively if inert core adsorbents are used. A decrease in the zone spreading time is important for desorption process, especially for biomacromolecule separation.

Using different core-radius fractions, numerical moments of component 1 and component 2 are plotted for Dirichlet BCs in Figures 8 and 9, respectively. In this case $c_{b_{1} \text {,inj }}=0.5 \mathrm{~g} / \mathrm{l}$, $c_{b_{2}, \text { inj }}=0.5 \mathrm{~g} / \mathrm{l}$ and $\omega=0.2$. The plots of zeroth moment shows that the total mass of component 1 increases on increasing the value of $\beta$. Whereas, the total mass of component 2 decreases on increasing $\beta$. It is observed that on increasing $\beta$ from 0 to 0.8 (fully porous beads to thin shell beads), the values of first, second and third moments are decreasing. The first moment $\mu_{1}$, characterizing the retention time of the elution profile, decreases on increasing the speed $u$ and it further decreases on increasing the value of $\beta$. The second central moment explains the variance (spreading) of the elution profiles. The concentration profiles become shaper for larger value of $\beta$ which indicates a decrease in the variance in Figures 8 and 9 for both components. Moreover, asymmetry and skewness of the elution profiles are explained by third moment, which is also decreasing on increasing the value of $\beta$ in Figures 8 and 9. Also, on increasing $\beta$ concentration profiles of both components are right-tailed.

Figures 10 and 11 display the first three numerical temporal moments of component 1 and 
component 2, respectively. All the moments are compared as functions of $B i_{p}$ for various core radius fractions $((\beta=0.0)$ to $(\beta=0.8))$ using dirichlet BCs. Plots of zeroth moment depict that mass of the reactant(Component 1) increases, while mass of the product decreases on increasing the value of core radius fraction from 0.0 to 0.8 . It also indicates that conversion of the reactant (Component 1) into the product (Component 2) decreases on increasing the value of $\beta$. Moreover, on increasing the core radius $\beta$ from 0.0 to 0.8 , the first, second and third moments of both component 1 and component 2 are decreasing. The figures also show that retention time, spreading and skewness of concentration profiles decreases on increasing $\beta$.

In Figures 12 and 13, the numerical moments of component 1 and component 2 are plotted versus intraparticle diffusion parameter $\eta$ for various core radius fractions $((\beta=0.0)$ to $(\beta=0.8)$. Once again, the magnitude of zeroth moment depicts a reduced conversion on increasing the value of core-radius fraction from 0.0 to 0.8 . Moreover, observations for the first, second and third moments are analogous to the above discussions.

\section{Problem 2: Linear case of Reversible reaction}

This section presents some numerical cases studies for reversible reactions. Both analytical and numerical solutions are in good agreement with each other showing accuracy of the numerical scheme and correctness of the analytical solutions. All the parameters used in the test problems are listed in Table 1.

Figure 14 shows concentration profiles for the core radius fraction $\beta=0.5$, when a finite width pulse is injected in an initially regenerated column $\left(c_{i, \text { init }}=0.0 \mathrm{~g} / \mathrm{l}\right.$ for $\left.i=1,2\right)$ using Dirichlet BCs. Here, the reaction rate constants are taken as $\omega_{1}=0.2$, and $\omega_{2}=0.1$.

In Figure 14(a) the amount of injection for both components is the same $\left(c_{b_{i}, \text { inj }}=0.5 \mathrm{~g} / \mathrm{l}\right.$ for $i=1,2)$, where as in Figure $14(\mathrm{~b}) c_{b_{1}, \text { inj }}=0.5 \mathrm{~g} / \mathrm{l}$ and $c_{b_{2}, \text { inj }}=0.25 \mathrm{~g} / \mathrm{l}$. The effects of injection for reversible reaction in Figures $14(\mathrm{a})$ and 14(b) are almost the same to 
irreversible reaction presented in Figures 2(a) and 2(b). Good agreements in the solution profiles validate correctness of the numerical Laplace inversion and accuracy of the HRFVS.

Finally, in Figures 15 and 16, the numerical temporal moments of semi-analytical solutions for dirichlet BCs are presented for different core radius fractions. For the case of reversible reaction and various flow speeds, the zeroth, first, 2nd central and third central moments are calculated for both components. The zeroth moments reflect the obvious effects of flow velocity on the conversion of reactant and it is consistently decreasing its value when $\beta$ is increased from 0.0 to 0.8. From the Figures $15(\mathrm{~b})$ and $16(\mathrm{~d})$, the first moment tells that on increasing the speed, retention time of component 1 (reactant) is higher than component 2 (product) and this retention time reduces on increasing $\beta$ for both components. The plots of second central moments reveal that variances (spreadings) of both components are reducing on increasing the value of $\beta$. Furthermore, the third central moments, which

depict asymmetry of concentration profiles, are also decreasing on increasing the value of $\beta$.

\section{Conclusion}

The semi-analytical solutions and numerical temporal moments were derived for the twocomponent GRM simulating linear reactive chromatography in fixed-bed columns packed with core-shell particles. The Laplace transformation, the eigen-decomposition, and the numerical back transformation techniques were utilized to derive the analytical solutions. The second order accurate finite volume scheme was applied to solve the model equations numerically. Moreover, the numerical temporal moments were obtained from the semianalytical solutions. Good matching of the analytical and numerical solutions verified accuracy of the suggested numerical scheme and helped in gaining confidence on the de- 
rived analytical solutions. Selected parametric studies for core-radius fractions, reaction rate constants, intraparticle diffusion resistance, film mass transfer resistance and axial dispersion were performed. The results showed that the column hold-up capacity decreases with an increase in the core radius fraction, which result in faster and sharper breakthrough curves. Also, the column efficiency increased due to reduced diffusional path when it is packed with inert core adsorbents. However, it was found that conversion of the reactants into product decreases on increasing the core radius fraction due to limited volume where the reaction is taking place. The derived and carefully validated solutions are seen as a useful tool to produce further optimized core-shell particles and to apply them efficiently

in chromatographic reactors. As stated above, the results explain and quantify both the advantages and disadvantages of using such particles that allow exploiting higher column efficiencies but reduced active volumes. The analysis further allows determining optimal operating conditions in terms of flow rate and injected amount for such particles.

\section{References}

(1) Kirkland, J. J.; Truszkowski, F. A.; Dilks, C. H.; Engel, G. S. Superficially Porous Silica Microspheres for Fast High-Performance Liquid Chromatography of Macromolecules. J. Chromatogr. A 2000, 890, 3.

(2) Coutinho, F. M. B.; Carvalho, D. L.; La Torre Aponte, M. L.; Barbosa, C. C. R. Pellicular Ion Exchange Resins Based on Divinylbenzene and 2-Vinylpyridine. Polymer 2001, 42, 43.

(3) Greibrokk, T. The Contributions of Csaba Horváth to Liquid Chromatography. J. Sep. Sci. 2004, 27, 1249. 
(4) Zhou, X.; Sun, Y.; Liu, Z. Superporous Pellicular Agarose-Glass Composite Particle for Protein Adsorption. Biochem. Eng. J. 2007, 34, 99.

(5) Manchón, N.; D’Arrigo, M.; García-Lafuente, A.; Guillamón, E.; Villares, A.; Ramos, A.; Martínez, J. A.; Rostagno, M. A. Fast Analysis of Isoflavones by High-Performance Liquid Chromatography Using a Column Packed With Fused-Core Particles. Talanta 2010, 82, 1986.

(6) Horvath, C. G.; Preiss, B. A.; Lipsky, S. R. Fast Liquid Chromatography. Investigation of Operating Parameters and the Separation of Nucleotides on Pellicular Ion Exchangers. Anal. Chem. 1967, 39, 1422.

(7) Guiochon, G.; Gritti, F. Shell Particles, Trials, Tribulations and Triumphs. J. Chromatogr. A 2011, 1218, 1915.

(8) Hayes, R.; Ahmed; A.; Edge, T.; Zhang, H. Core-Shell Particles: Preparation, Fundamentals and Applications in High Performance Liquid Chromatography. J. Chromatogr. A 2014, 1357, 36.

(9) Lee, W.-C. Protein Separation Using Non-Porous Sorbents. J Chromatogr. B 1997, 699, 29.

(10) Rissler, K. Separation of Polyesters by Gradient Reversed-Phase High-Performance Liquid Chromatography on a $1.5 \mu \mathrm{m}$ Non-Porous Column. J. Chromatogr. A 2000, $871,243$.

(11) Xiang, Y; Yan, B.; McNeff, C. V.; Carr, P. W.; Lee, M. L. Synthesis of Micron Diameter Polybutadiene-Encapsulated Non-Porous Zirconia Particles for Ultrahigh Pressure Liquid Chromatography. J. Chromatogr. A 2003, 1002, 71. 
(12) Fekete, S.; Ganzler, K.; Fekete, J. Facts and Myths About Columns Packed With sub-3 $\mu m$ and sub-2 $\mu m$ Particles. J. Pharm. Biomed. Anal. 2010, 51, 56.

(13) Gu, T.; Liu, M.; Cheng, K.-S. C.; Ramaswamy, S.; Wang, C. A General Rate Model Approach for the Optimization of the Core Radius Fraction for Multicomponent Isocratic Elution in Preparative Nonlinear Liquid Chromatography Using Cored Beads. Chem. Eng. Sci. 2011, 66, 3531.

(14) Miyabe, K. Evaluation of Chromatographic Performance of Various Packing Materials Having Different Structural Characteristics as Stationary Phase for Fast High Performance Liquid Chromatography by New Moment Equations. J. Chromatogr. A 2008, 1183, 49 .

(15) Miyabe, K. Moment Equations for Chromatography Based on Langmuir Type Reaction Kinetics. J. Chromatogr. A 2014, 1356, 171.

(16) Miyabe, K. Moment Theory for Kinetic Study of Chromatography. Trends Anal. Chem. 2016, 81, 79.

(17) Ding, Y.; Sun, Y. Small-Sized Dense Magnetic Pellicular Support for Magnetically Stabilized Fluidized Bed Adsorption of Protein. Chem. Eng. Sci. 2005, 60, 917.

(18) Wang, X. L.; Barber, W. E.; Carr, P. W. A Practical Approach to Maximizing Peak Capacity by Using Long Columns Packed With Pellicular Stationary Phases for Proteomic Research. J. Chromatogr. A 2006, 110\%, 139.

(19) Kirkland, J. J. Controlled Surface Porosity Supports for High-Speed Gas and Liquid Chromatography. Anal. Chem. 1969, 41, 218.

(20) Pesek, J. J.; Frost, J. H. Adsorption as a Mechanism for Separation of Nonionic Solutes by Pellicular Ion Exchange Chromatography. Anal. Chem. 1973, 45, 1762. 
(21) Ning, J.; Kong, F. Z.; Li, D. H.; Du, Y. Z. Preparation of Monodisperse Agglomerated Pellicular Anion-Exchange Resins Compatible With High-Performance Liquid Chromatography Solvents for Ion Chromatography. J. Chromatogr. A 1973, 793, 193.

(22) Kiss, I.; Bacskay, I., Kilár, F.; Felinger, A. Comparison of the Mass Transfer in Totally Porous and Superficially Porous Stationary Phases in Liquid Chromatography. Anal. Bioanal. Chem. 2010, 397, 1307.

(23) Cabooter, D.; Fanigliulo, A.; Bellazzi, G.; Allieri, B.; Rottigni, A.; Desmet, G. Relationship Between the Particle Size Distribution of Commercial Fully Porous and Superficially Porous High-Performance Liquid Chromatography Column Packings and Their Chromatographic Performance. J. Chromatogr. A 2010, 1217, 7074.

(24) Fanigliulo, A.; Cabooter, D.; Bellazzi, G.; Tramarin, D.; Allieri, B.; Rottigni, A.; Desmet, G. Comparison of Performance of High Performance Liquid Chromatography Columns Packed With Superficially and Fully Porous $2.5 \mu \mathrm{m}$ Particles Using Kinetic Plots. J. Sep. Sci. 2010, 33, 3655.

(25) Kaczmarski, K.; Guiochon, G. Modeling of the Mass-Transfer Kinetics in Chromatographic Columns Packed With Shell and Pellicular Particles. Anal. Chem. 2007, 79, 4648.

(26) Kaczmarski, K. On the Optimization of the Solid Core Radius of Superficially Porous Particles for Finite Adsorption Rate. J. Chromatogr. A 2010, 1218, 951.

(27) Li, P.; Yu, J.; Xiu, G.; Rodrigues, A. E. A Strategy for Tailored Design of Efficient and Low-Pressure Drop Packed Column Chromatography. AIChE J. 2010, 56, 3091.

(28) Lambert, N.; Kiss, I.; Felinger, A. Significance and Estimation of Chromatographic Parameters. J. Chromatogr. A 2014, 1366, 84. 
(29) Qamar, S.; Abbasi, J. N.; Mehwish, A.; Seidel-Morgenstern, A. Linear General Rate Model of Chromatography for Core-Shell Particles: Analytical Solutions and Moment Analysis. Chem. Eng. Sci. 2015, 137, 352.

(30) Qamar, S.; Sattar, F. A.; Abbasi, J. N.; Seidel-Morgenstern, A. Numerical Simulation of Nonlinear Chromatography With Core-Shell Particles Applying the General Rate Model. Chem. Eng. Sci. 2016, 14\%, 54.

(31) Ganetsos, G.; Barker, P. E. Preparative and Production Scale Chromatography. Chromatographic Science Series: Vol. 61; Marcel Dekker, Inc.: New York, 1993; p 375.

(32) Guiochon, G.; Felinger, A.; Shirazi, D. G.; Katti, A. M. Fundamentals of Preparative and Nonlinear Chromatography; 2nd ed; ELsevier Academic press: New York, 2006.

(33) Lin, B.; Song, F.; Guiochon, G. Analytical Solution of the Ideal, Nonlinear Model of Reaction Chromatography for a Reaction $\mathrm{A} \rightarrow \mathrm{B}$ and a Parabolic Isotherm. J. Chromatogr. A 2003, $1003,91$.

(34) Ruthven, D. M. Principles of Adsorption and Adsorption Processes; WileyInterscience: New York, 1984.

(35) Carta, G. Simultaneous Reaction and Chromatography. In Chromatographic and Membrane Processes in Biotechnology; Costa, C. A., Cabral, J. S., Eds.; Kluwer Academic Publishers: Dordrecht, The Netherlands, 1991; p 429.

(36) Schweich, D.; Villermaux, J. The chromatographic Reactor. A New Theoretical Approach. Ind. Eng. Chem. Fundamentals 1978, 17, 1.

(37) Cho, B. K.; Aris, R.; Carr, R. W. The Mathematical Theory of a Countercurrent Catalytic Reactor. Proc. R. Soc. London, Ser. A 1982, 383, 147. 
(38) Petroulas, T.; Aris, R.; Carr, R. W. Analysis of the Counter-Current Moving-Bed Chromatographic Reactor. Comp. \& Maths. with Appls. 1985, 11, 5.

(39) Takeuchi, K.; Uraguchi, Y. Separation Conditions of the Reactant and the Product With a Chromatographic Moving Bed Reactor. J. Chem. Eng. Jpn. 1976, 9, 164.

(40) Takeuchi, K.; Uraguchi, Y. Basic Design of Chromatographic Moving-Bed Reactors for Product Refining. J. Chem. Eng. Jpn. 1976, 9, 246.

(41) Takeuchi, K., Uraguchi, Y. The Effect of the Exhausting Section on the Performance of a Chromatographic Moving-Bed Reactor. J. Chem. Eng. Jpn. 1977, 10, 72.

(42) Takeuchi, K.; Uraguchi, Y. Experimental Studies of a Chromatographic Moving-Bed Reactor-Catalytic Oxidation of Carbon Monoxide on Activated Alumina as a Model Reactor. J. Chem. Eng. Jpn. 1977, 10, 455.

(43) Takeuchi, K.; Miyauchi, T.; Uraguchi, Y. Computational Studies of a Chromatographic Moving Bed Reactor for Consecutive and Reversible Reactions. J. Chem. Eng. Jpn. 1978, 11, 216.

(44) Binous, H.; McCoy, B. Chromatographic Reactions of the Three Components: Application to Separations. Chem. Eng. Sci. 1992, 47, 4333.

(45) Borren, T., Fricke, J., Schmidt-Traub, H., Eds. Chromatographic Reactors in Preparative Chromatography of Fine Chemicals and Pharmaceutical Agents; Wiley-VCH Verlag: Weinheim, Germany, 2005; p 371.

(46) Fricke, J.; Schmidt-Traub, H.; Kawase, M. Chromatographic Reactor, Ullmann's Encyclopedia of Industrial Chemistry; Wiley-VCH Verlag: Weinheim, 2005. 
(47) Javeed, S.; Qamar, S.; Seidel-Morgenstern, A.; Warnecke, G. A Discontinuous Galerkin Method to Solve Chromatographic Models. J. Chromatogr. A 2011, 1218, 7137.

(48) Yamaoka, K.; Nakagawa, T. Moment Analysis for Reaction Chromatography. J. Chromatogr. A 1976, 117, 1.

(49) Sardin, M., Schweich, D., Villermaux, J., Ganetsos, G., Barker, P. E., Eds. Preparative Fixed-Bed Chromatographic Reactor, Preparative and Production Scale Chromatography; Marcel Dekker Inc.: New York, 1993; p 477.

(50) Villermaux, J., Rodrigues, A. E., Tondeur, D., Eds. The Chromatographic Reactor in Percolation Processes: Theory and application; Sijthoff and Noordhoff: Alpena an den Rijn, The Netherlands, 1981; p 539.

(51) Javeed, S.; Qamar, S.; Seidel-Morgenstern, A.; Warnecke, G. Parametric Study of Thermal Effects in Reactive Liquid Chromatography. Chem. Eng. J. 2012, 191, 426.

(52) Qamar, S.; Bibi, S.; Khan, F. U.; Shah, M.; Javeed, s.; Seidel-Morgenstern, A. Irreversible and Reversible Reactions in a Liquid Chromatographic Column: Analytical Solutions and Moment Analysis. Ind. Eng. Chem. Res. 2014, 53, 2461.

(53) Bibi, S.; Qamar, S.; Seidel-Morgenstern, A. Irreversible and Reversible Reactive Chromatography: Analytical Solutions and Moment Analysis for Rectangular Pulse Injections. J. Chromatogr. A 2015, 1385, 49.

(54) Qamar, S.; Bibi, S.; Akram, N.; Seidel-Morgenstern, A. Analysis of Two-Component Non-Equilibrium Model of Linear Reactive Chromatography, Chromatogrpahia 2017, 80,383 . 
(55) Bashir, S.; Qamar, S.; Perveen, S.; Seidel-Morgenstern, A. Analysis of Linear Reactive General Rate Model of Chromatography Considering Irreversible and Reversible Reactions. Chem. Eng. Res. Des. 2017, 119, 140.

(56) Qamar, S., Sattar, F. A., Seidel-Morgenstern, A., 2016. Theoretical Investigation of Thermal Effects in Non-Isothermal Non-Equilibrium Reactive Liquid Chromatography, Chem. Eng. Res. Des. 115, 145.

(57) Rice, R. G.; Do, D. D. Applied Mathematics and Modeling for Chemical Engineers; Wiley-Interscience: New York, 1995.

(58) Quezada, C. R.; Clement, T. P.; Lee, K. K. Generalized Solution to Multi-Dimensional Multi-Species Transport Equations Coupled With a First-Order Reaction Network Involving Distinct Retardation Factors. Adv. Water Resour. 2004, 27, 507.

(59) Durbin, F. Numerical Inversion of Laplace Transforms: An Efficient Improvement to Dubner and Abate's Method. The Computer J. 1974, 17, 371.

(60) Javeed, S.; Qamar, S.; Seidel-Morgenstern, A.; Warnecke, G. Efficient and Accurate Numerical Simulation of Nonlinear Chromatographic Processes. J. Comput. Chem. Eng. 2011, 35, 2294.

(61) Koren, B. A Robust Upwind Discretization Method for Advection, Diffusion and Source Terms. In Numerical Methods for Advection-Diffusion Problems, Vol. 45 of Notes on Numerical Fluid Mechanics; Vreugdenhil, C. B., Koren, B., Eds.; Vieweg Verlag: Braunschweig, Germany, 1993; Chapter 5, p 117.

(62) Danckwerts, P.V. Continuous Flow Systems. Chem. Eng. Sci. 1953, 2, 1. 


\section{List of Figures}

1 Schematic diagrams of fixed-bed reactor and inert core adsorbent. . . . . .

2 Irreversible reaction: Influence of the volume of injection of product on the concentrations profiles obtained by Dirichlet BCs at $x=1$ for $t_{\text {inj }}=10 \mathrm{~min}$.

3 Irreversible reaction: Influence of core-radius fraction $\left(\beta=R_{\text {core }} / R_{p}\right)$ on the concentration profiles obtained by Dirichlet BCs at $x=1$ for $\omega_{1}=0.2$, $t_{\text {inj }}=10 \mathrm{~min}$ and $c_{b_{i} \text {,inj }}=0.5 \mathrm{~g} / l(i=1,2)$. Plots (a) and (b): $D_{b}=0.34$ or $P e_{b}=\frac{L u}{D_{b}}=73.53$, plots (c) and (d): $D_{b}=0.0034$ or $P e_{b}=7353 \ldots . .$.

4 Irreversible reaction: Effects of Dirichlet and Danckwerts BCs on the concentration profiles at $x=1$. Here, $c_{b_{i}, \text { inj }}=0.5 \mathrm{~g} / l(i=1,2), t_{\mathrm{inj}}=10 \mathrm{~min}$ and $\omega_{1}=0.2 \ldots \ldots \ldots \ldots \ldots$

$5 \quad$ Irreversible reaction: Effects of dimensionless forward reaction rate constant $\omega_{1}$ on the concentration profiles at $x=1$, using Danckwerts BCs. Here, $c_{b_{i}, \text { inj }}=0.5 \mathrm{~g} / l(i=1,2), t_{\text {inj }}=10$ min and $\omega_{1}=0.2 \ldots \ldots$

6 Irreversible reaction: Influence of $B i_{p}=\frac{k_{\operatorname{ext}_{i} R_{p}}}{D_{\text {eff }_{\mathrm{i}}}}$ on the concentration profiles for $c_{b_{i}, \text { inj }}=0.5 \mathrm{~g} / l(i=1,2), t_{\mathrm{inj}}=10 \mathrm{~min}$ and $\omega_{1}=0.2 \ldots \ldots$

7 Irreversible reaction: Influence of $\eta_{p}$ on the concentration profiles for $c_{b_{i} \text {,inj }}=$ $0.5 \mathrm{~g} / l(i=1,2), t_{\mathrm{inj}}=10 \mathrm{~min}$ and $\omega_{1}=0.2 \ldots \ldots . . . .$.

8 Irreversible reaction: Plots of average central moments (numerical) for component1 considering different flow-rates using Dirichlet BCs. Here, $c_{b_{i}, \text { inj }}=$ $0.5 \mathrm{~g} / l(i=1,2), t_{\mathrm{inj}}=10 \mathrm{~min}$ and $\omega_{1}=0.2 \ldots \ldots . . . .$.

9 Irreversible reaction: Plots of average central moments (numerical) of component2 considering different flow-rates using Dirichlet BCs. Here, $c_{b_{i}, \text { inj }}=$ $0.5 \mathrm{~g} / l(i=1,2), t_{\mathrm{inj}}=10 \mathrm{~min}$ and $\omega_{1}=0.2 \ldots \ldots . . . . .$. 
10 Irreversible reaction: Plots of average central moments (numerical) for component 1 considering different Biot numbers using Dirichlet BCs. Here, $c_{b_{i}, \text { inj }}=0.5 \mathrm{~g} / l(i=1,2), t_{\text {inj }}=10 \mathrm{~min}$ and $\omega_{1}=0.2 \ldots \ldots$.

11 Irreversible reaction: Plots of average central moments (numerical) for component 2 considering different Biot numbers using Dirichlet BCs. Here, $c_{b_{i}, \text { inj }}=0.5 \mathrm{~g} / l(i=1,2), t_{\text {inj }}=10 \mathrm{~min}$ and $\omega_{1}=0.2 \ldots \ldots$

12 Irreversible reaction: Plots of average central moments (numerical) for component 1 considering different values of $\eta_{p}$ using Dirichlet BCs. Here, $c_{b_{i}, \text { inj }}=$

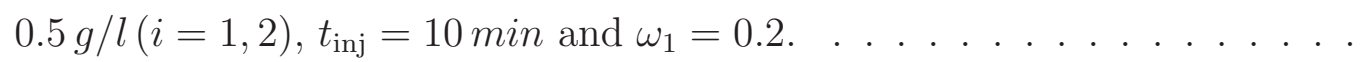

13 Irreversible reaction: Plots of average central moments (numerical) for component 2 considering different values of $\eta_{p}$ using Dirichlet BCs. Here, $c_{b_{i}, \text { inj }}=$ $0.5 \mathrm{~g} / l(i=1,2), t_{\mathrm{inj}}=10 \mathrm{~min}$ and $\omega_{1}=0.2 \ldots \ldots \ldots$

14 Reversible reaction: Influence of injection on the concentration profiles at $x=1$. Here, $c_{b_{i} \text {,inj }}=0.5 \mathrm{~g} / l(i=1,2), t_{\text {inj }}=10 \mathrm{~min}, \omega_{1}=0.2$ and $\omega_{2}=0.1$.

15 Reversible reaction: Plots of average central moments (numerical) of component1 considering different flow-rates using Dirichlet BCs. Here, Here, $c_{b_{i}, \mathrm{inj}}=0.5 \mathrm{~g} / \mathrm{l}(i=1,2), t_{\mathrm{inj}}=10 \mathrm{~min}, \omega_{1}=0.2$ and $\omega_{2}=0.1 \ldots \ldots$

16 Reversible reaction: Plots of average central moments (numerical) of component2 considering different flow-rates using Dirichlet BCs. Here, Here, $c_{b_{i}, \mathrm{inj}}=0.5 \mathrm{~g} / \mathrm{l}(i=1,2), t_{\mathrm{inj}}=10 \mathrm{~min}, \omega_{1}=0.2$ and $\omega_{2}=0.1 \ldots \ldots$ 
Table 1: Parameters of linear GRM for reactive chromatography.

\begin{tabular}{|c|c|}
\hline Parameters & values \\
\hline Column length & $L=10 \mathrm{~cm}$ \\
external porosity & $\epsilon_{b}=0.4$ \\
internal porosity & $\epsilon_{p}=0.333$ \\
Interstitial velocity & $u=2.5 \mathrm{~cm} / \mathrm{min}$ \\
Axial dispersion coefficient & $D_{b}=0.34 \mathrm{~cm}^{2} / \mathrm{min}$ \\
Effective diffusivity coefficient & $D_{\text {eff }_{i}}=10^{-4} \mathrm{~cm}^{2} / \mathrm{min}(i=1,2)$ \\
Radius of particle & $R_{p}=4 \times 10^{-4} \mathrm{~cm}$ \\
External mass transfer coefficient & $k_{\mathrm{ext}_{i}}=0.01 \mathrm{~cm} / \mathrm{min}(i=1,2)$ \\
Time of injection & $t_{\text {inj }}=10 \mathrm{~min}$ \\
Total simulation time & $t_{\text {max }}=50 \mathrm{~min}$ \\
Inlet concentration & $c_{b_{i}, \text { inj }}=0.5 \mathrm{~g} / l(i=1,2)$ \\
$a_{1}=2.5$ \\
Adsorption equilibrium constant for component 1 \\
Adsorption equilibrium constant for component 2 \\
Dimensionless rate constant of forward reaction \\
Dimensionless rate constant of backward reaction
\end{tabular}

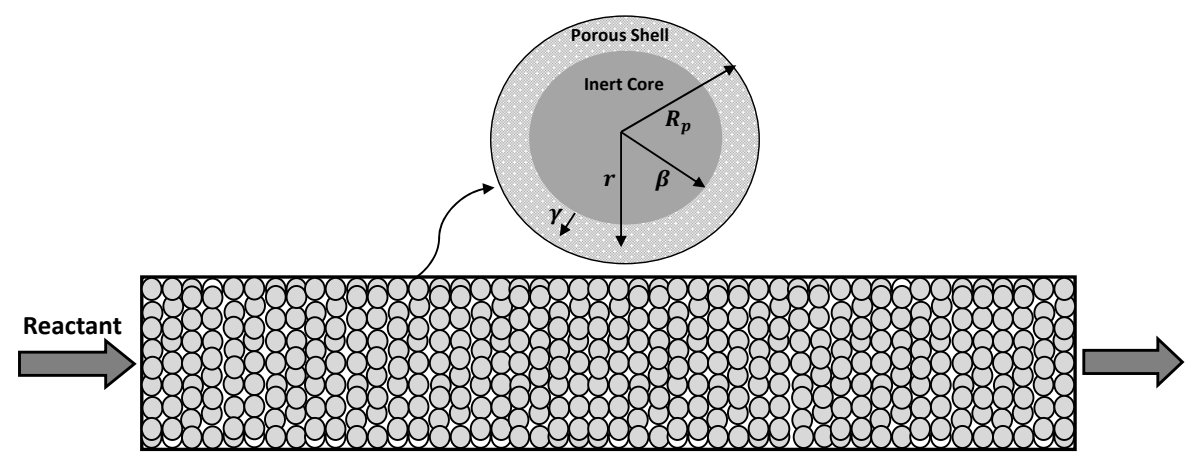

Figure 1: Schematic diagrams of fixed-bed reactor and inert core adsorbent. 

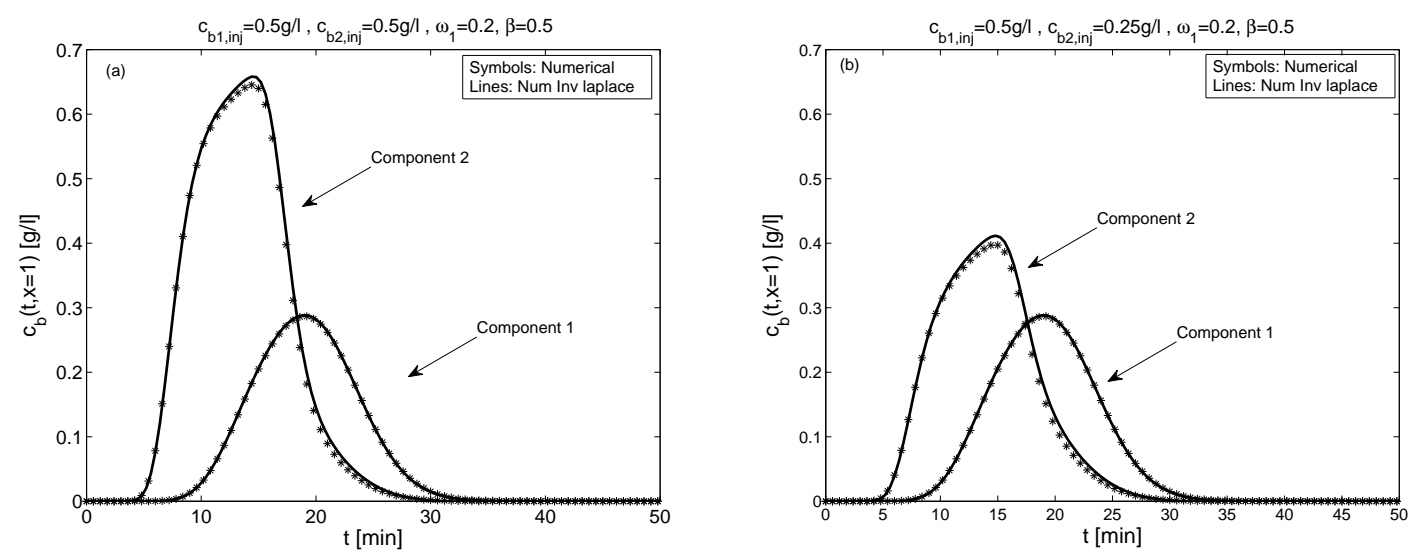

Figure 2: Irreversible reaction: Influence of the volume of injection of product on the concentrations profiles obtained by Dirichlet BCs at $x=1$ for $t_{\mathrm{inj}}=10 \mathrm{~min}$. 

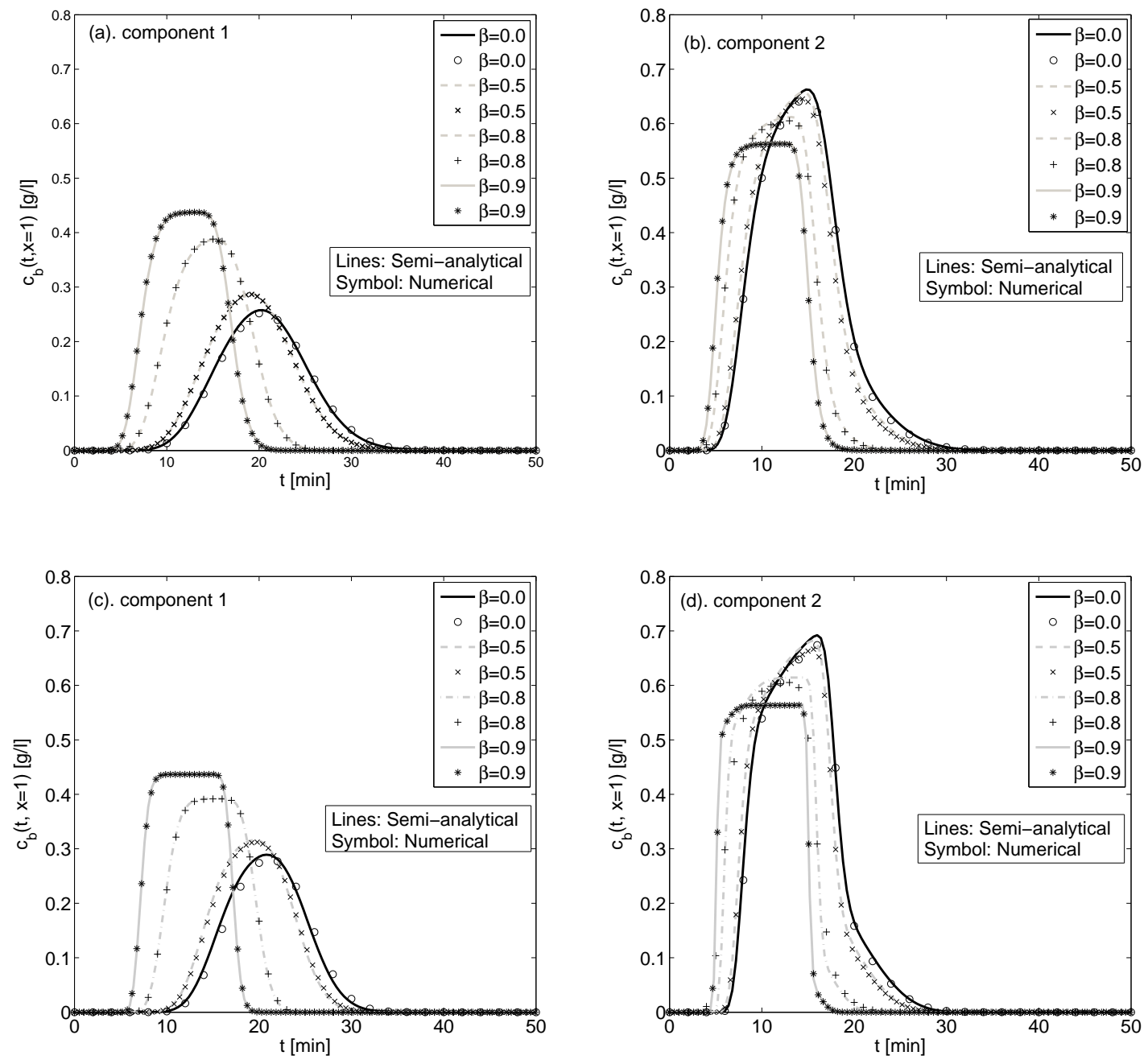

Figure 3: Irreversible reaction: Influence of core-radius fraction $\left(\beta=R_{\text {core }} / R_{p}\right)$ on the concentration profiles obtained by Dirichlet BCs at $x=1$ for $\omega_{1}=0.2, t_{\text {inj }}=10 \mathrm{~min}$ and $c_{b_{i}, \text { inj }}=0.5 \mathrm{~g} / l(i=1,2)$. Plots (a) and (b): $D_{b}=0.34$ or $P e_{b}=\frac{L u}{D_{b}}=73.53$, plots (c) and (d): $D_{b}=0.0034$ or $P e_{b}=7353$. 

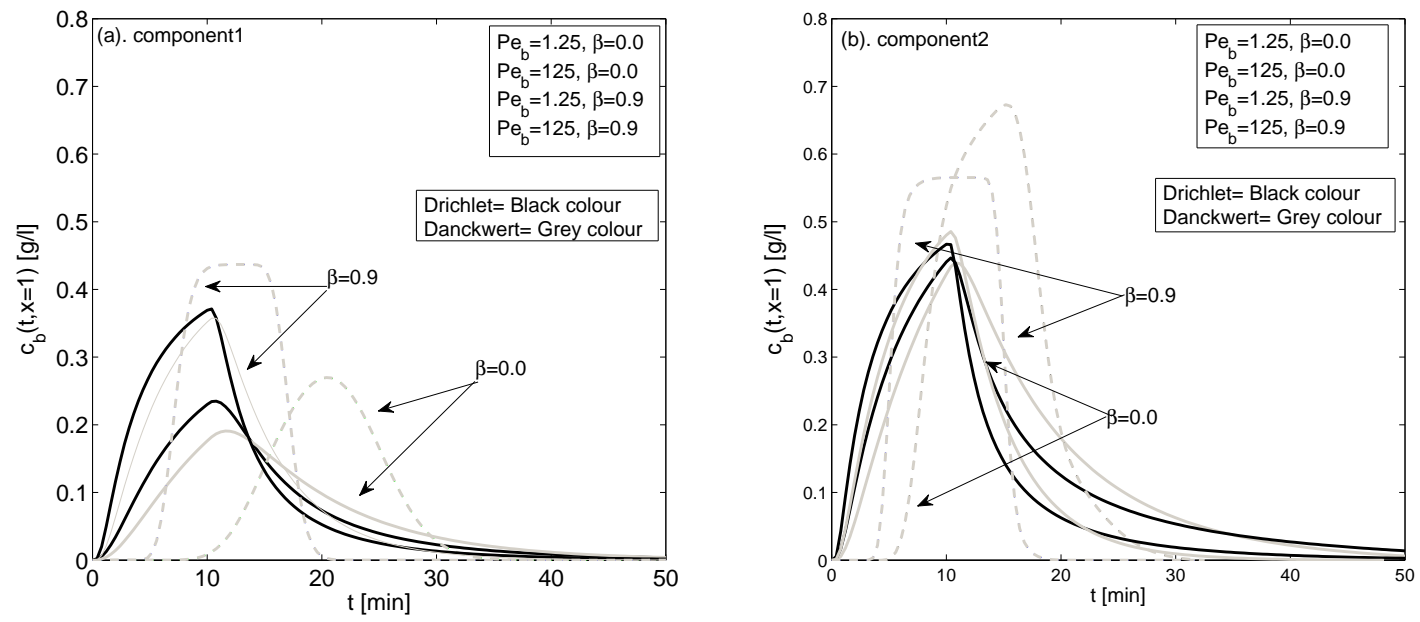

Figure 4: Irreversible reaction: Effects of Dirichlet and Danckwerts BCs on the concentration profiles at $x=1$. Here, $c_{b_{i} \text {,inj }}=0.5 \mathrm{~g} / l(i=1,2), t_{\mathrm{inj}}=10 \mathrm{~min}$ and $\omega_{1}=0.2$.
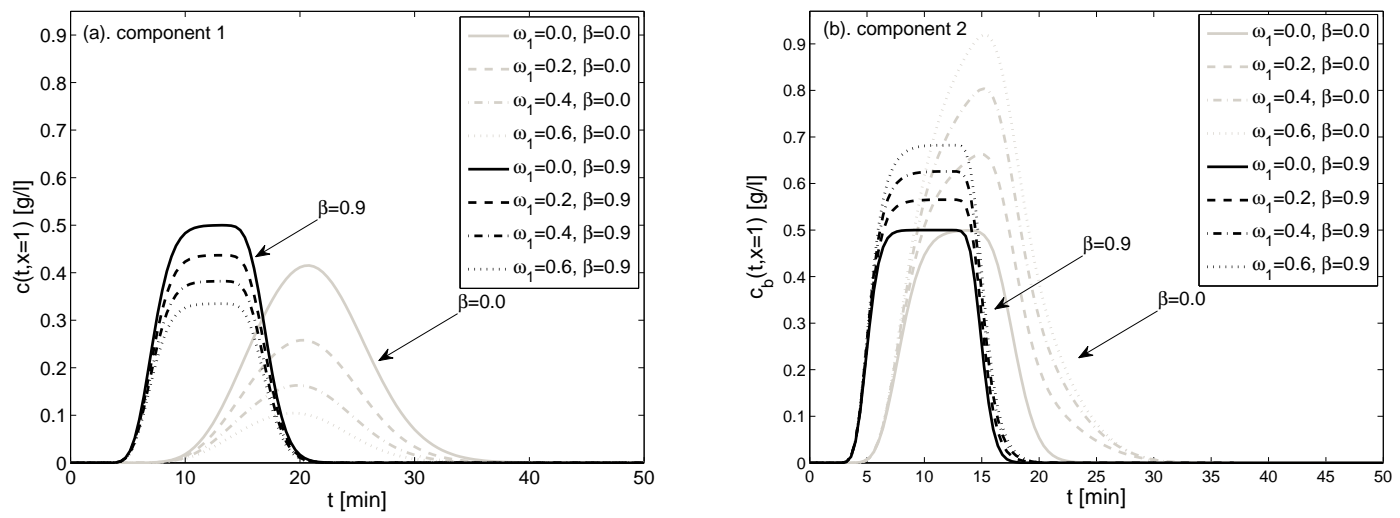

Figure 5: Irreversible reaction: Effects of dimensionless forward reaction rate constant $\omega_{1}$ on the concentration profiles at $x=1$, using Danckwerts BCs. Here, $c_{b_{i} \text {,inj }}=0.5 \mathrm{~g} / l(i=$ $1,2), t_{\text {inj }}=10 \mathrm{~min}$ and $\omega_{1}=0.2$. 

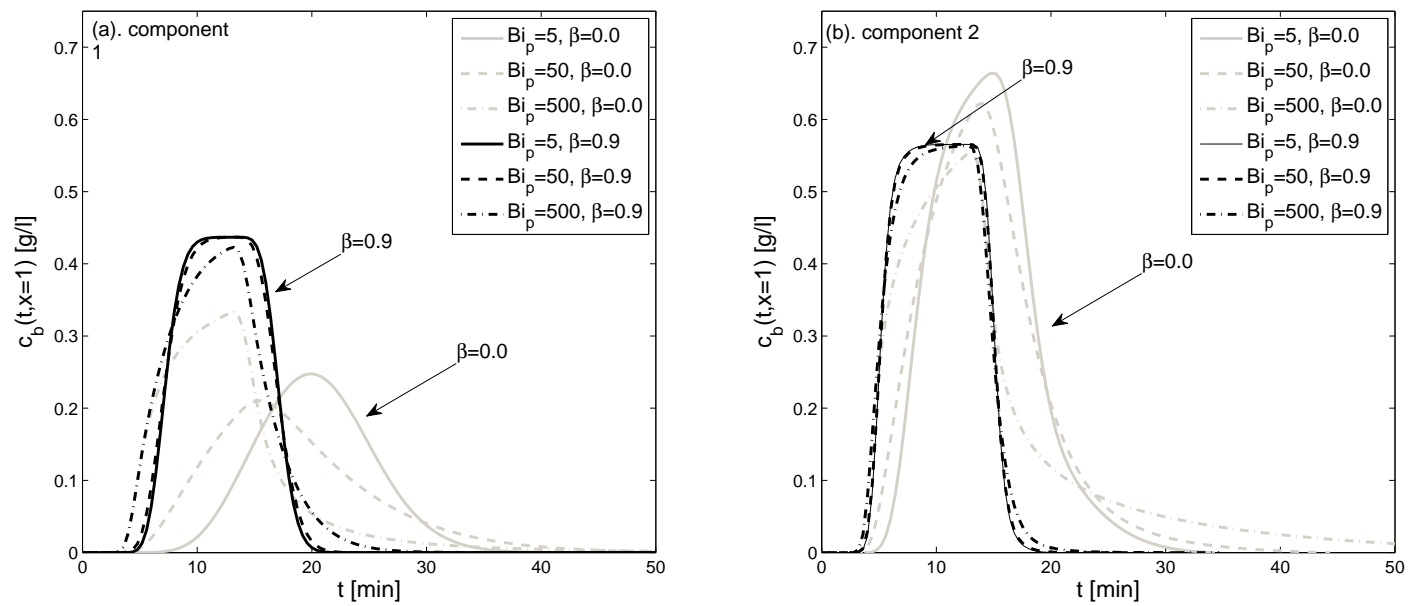

Figure 6: Irreversible reaction: Influence of $B i_{p}=\frac{k_{\text {ext }_{i} R_{p}}}{D_{\text {eff }}}$ on the concentration profiles for $c_{b_{i}, \text { inj }}=0.5 \mathrm{~g} / l(i=1,2), t_{\mathrm{inj}}=10 \mathrm{~min}$ and $\omega_{1}=0.2$.
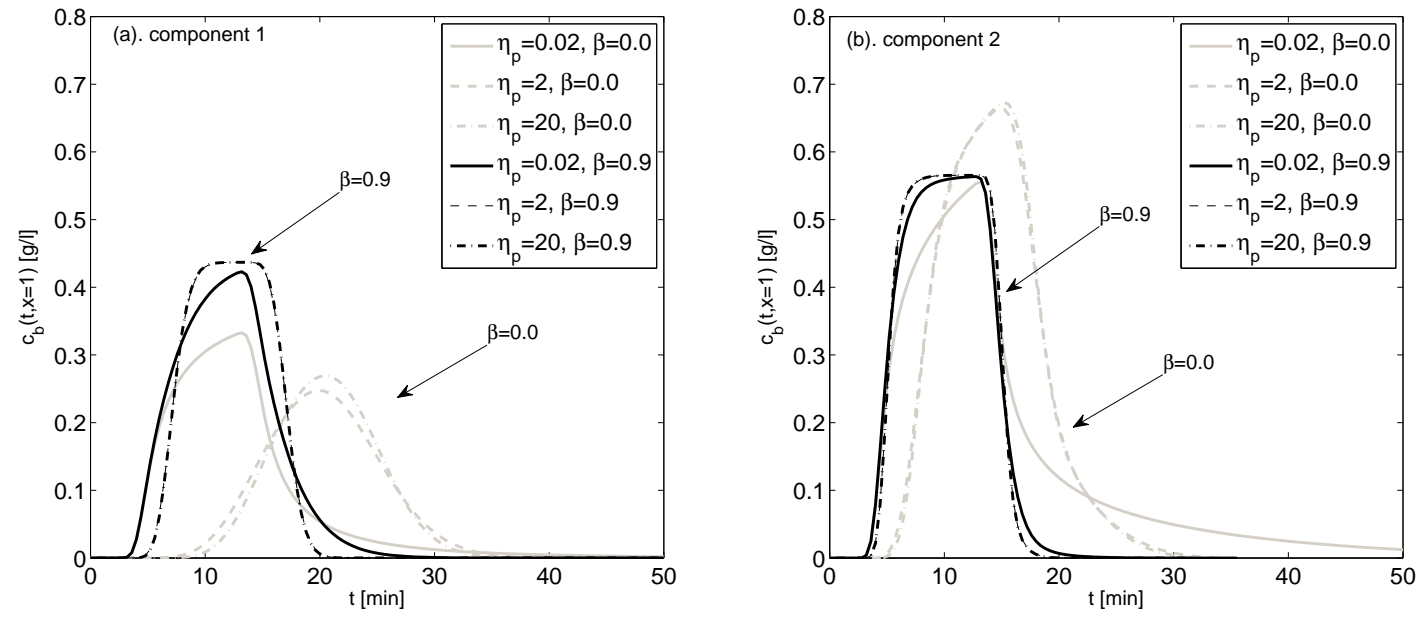

Figure 7: Irreversible reaction: Influence of $\eta_{p}$ on the concentration profiles for $c_{b_{i}, \text { inj }}=$ $0.5 \mathrm{~g} / \mathrm{l}(i=1,2), t_{\mathrm{inj}}=10 \mathrm{~min}$ and $\omega_{1}=0.2$. 

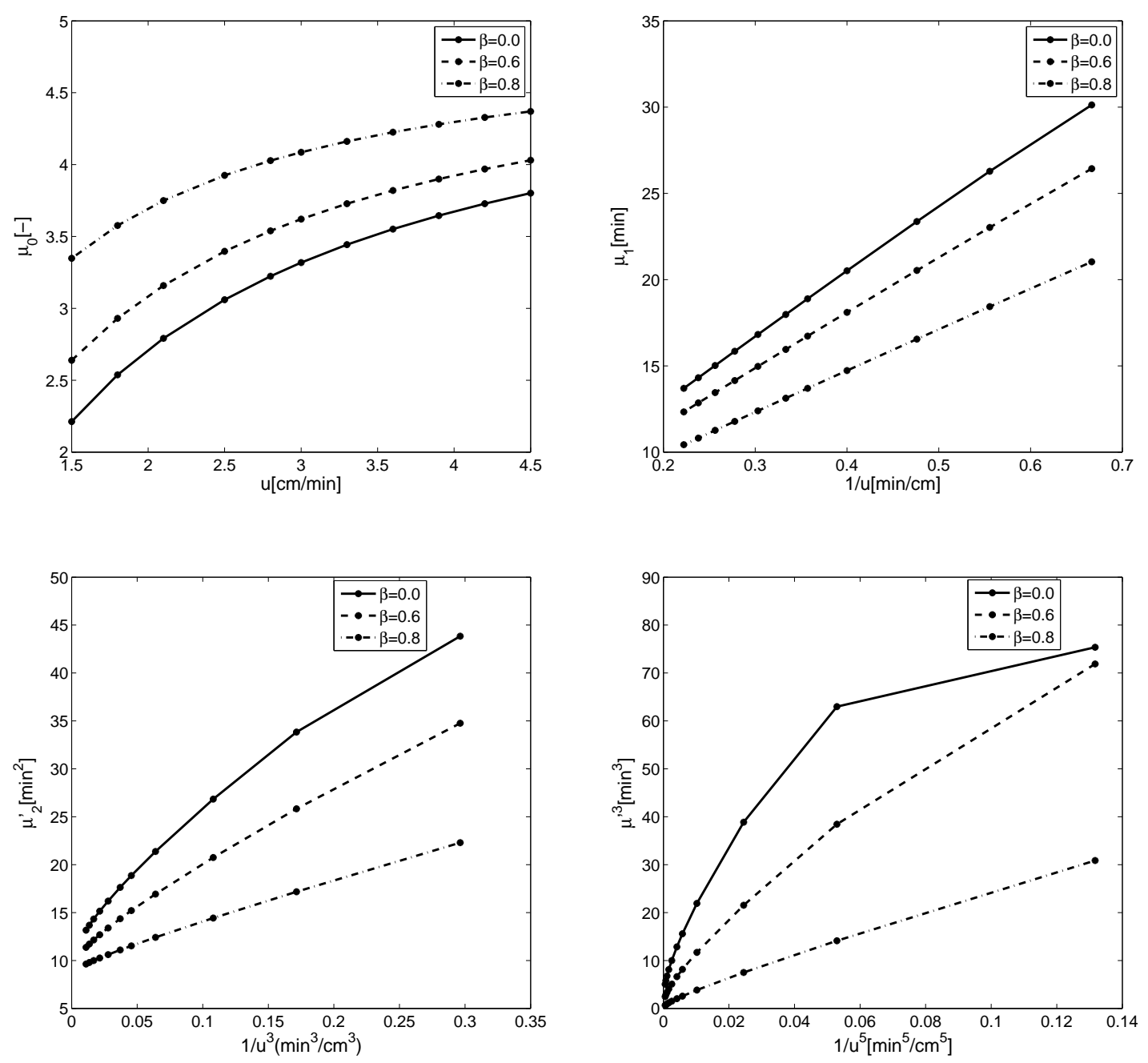

Figure 8: Irreversible reaction: Plots of average central moments (numerical) for component1 considering different flow-rates using Dirichlet BCs. Here, $c_{b_{i} \text {,inj }}=0.5 \mathrm{~g} / l(i=1,2)$, $t_{\text {inj }}=10 \min$ and $\omega_{1}=0.2$. 

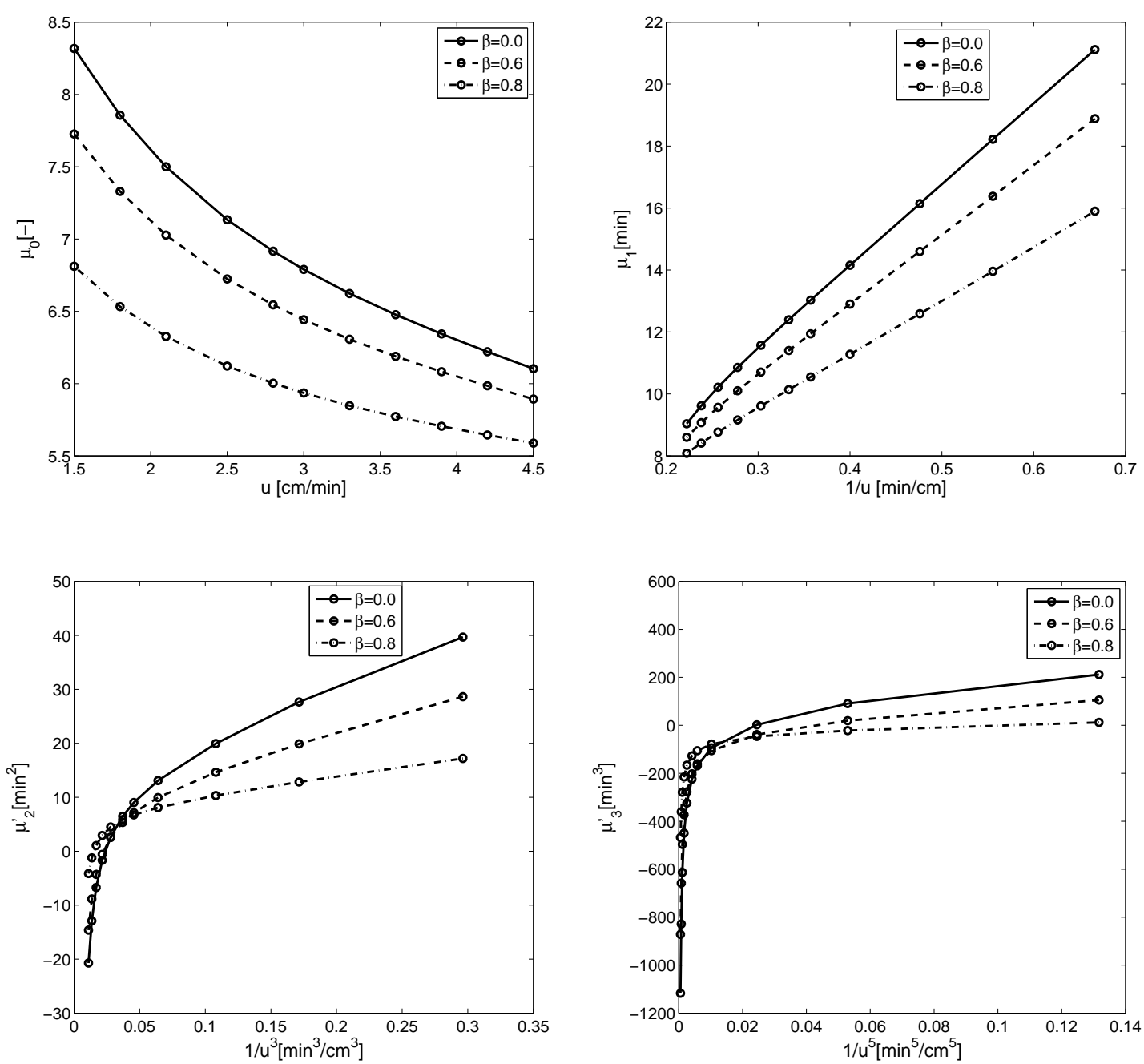

Figure 9: Irreversible reaction: Plots of average central moments (numerical) of component2 considering different flow-rates using Dirichlet BCs. Here, $c_{b_{i}, \text { inj }}=0.5 \mathrm{~g} / \mathrm{l}(i=1,2)$, $t_{\text {inj }}=10 \min$ and $\omega_{1}=0.2$. 

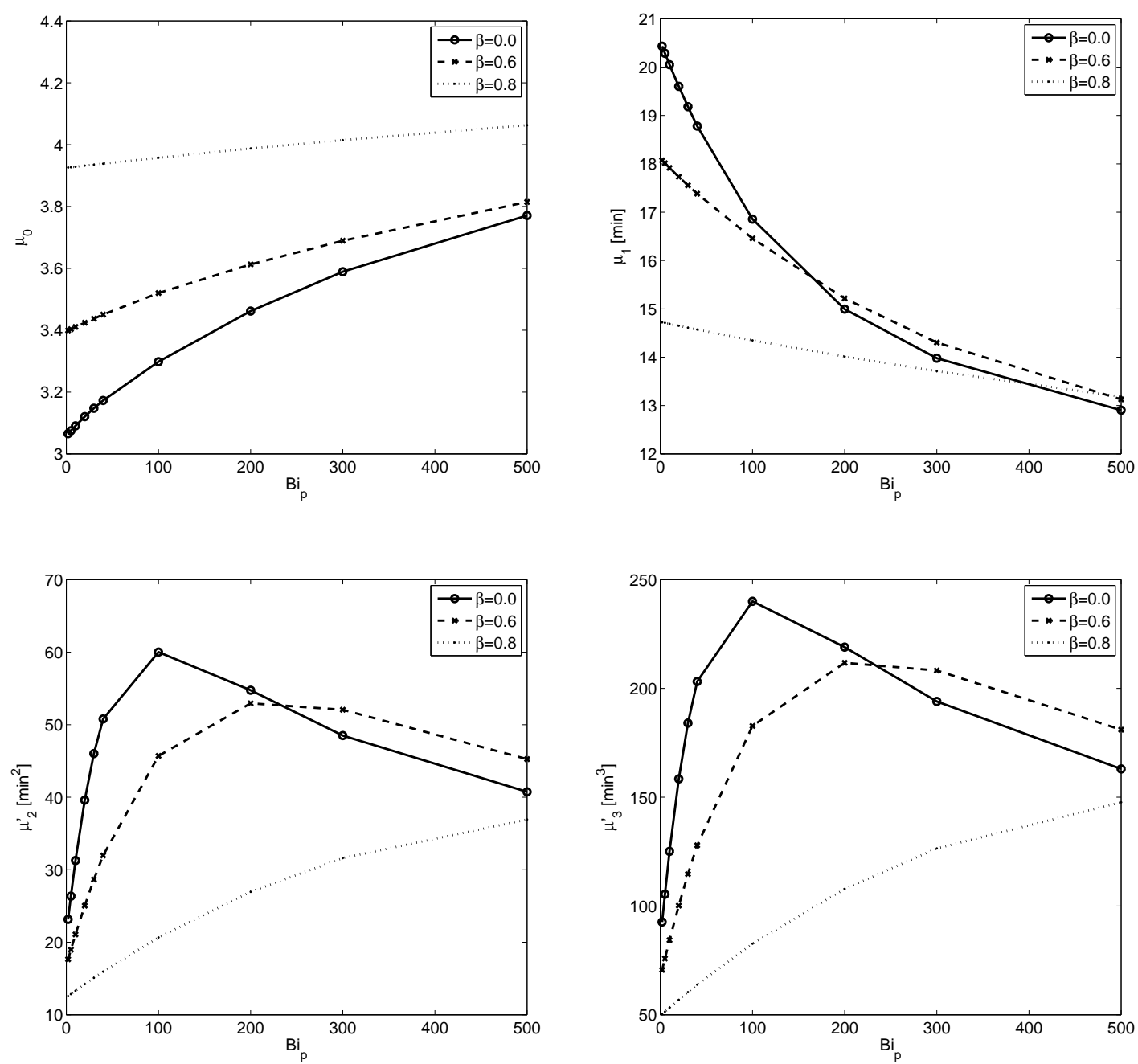

Figure 10: Irreversible reaction: Plots of average central moments (numerical) for component 1 considering different Biot numbers using Dirichlet BCs. Here, $c_{b_{i} \text {,inj }}=0.5 \mathrm{~g} / \mathrm{l}(i=$ $1,2), t_{\text {inj }}=10$ min and $\omega_{1}=0.2$. 

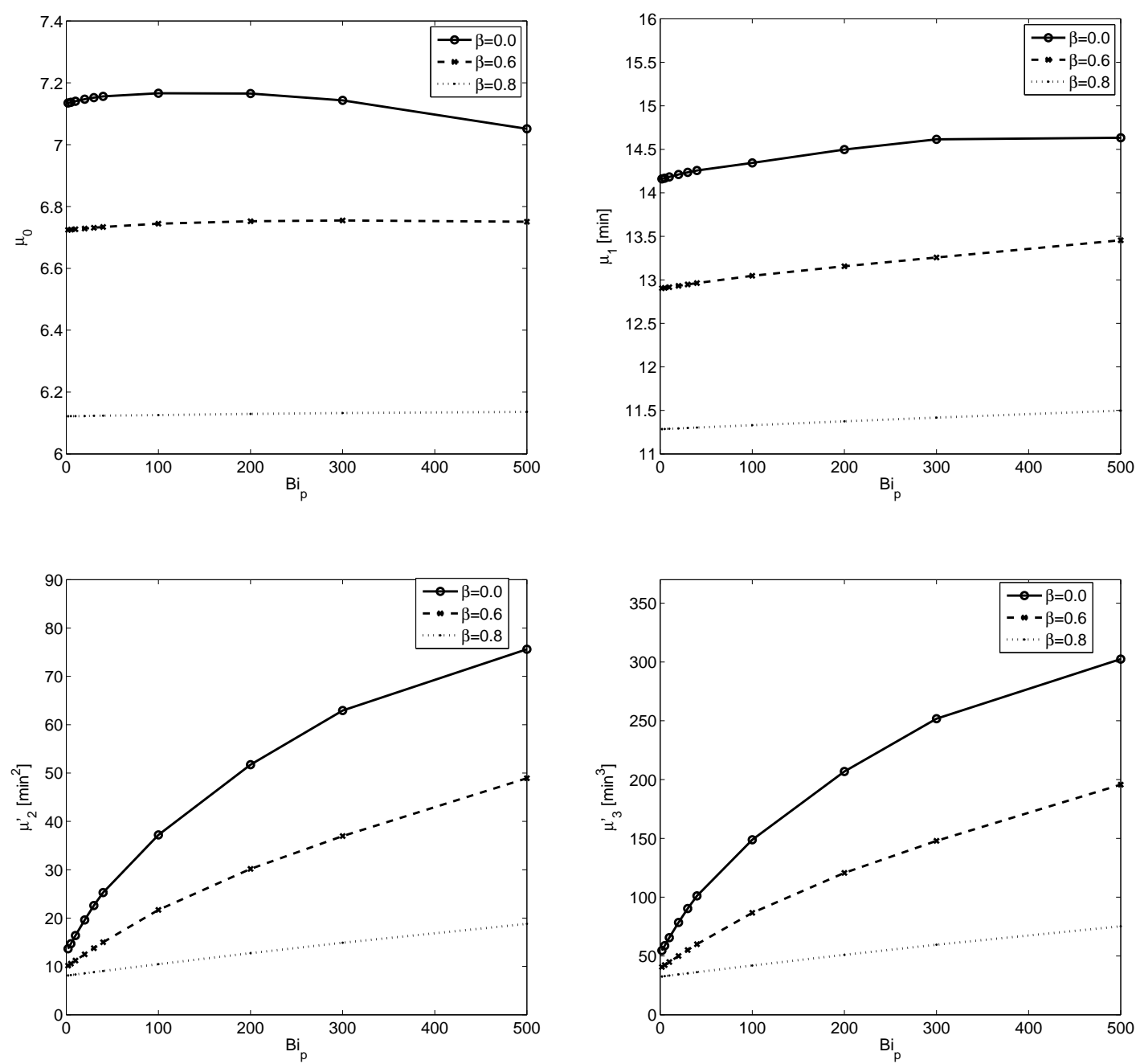

Figure 11: Irreversible reaction: Plots of average central moments (numerical) for component 2 considering different Biot numbers using Dirichlet BCs. Here, $c_{b_{i} \text {,inj }}=0.5 \mathrm{~g} / \mathrm{l}(i=$ $1,2), t_{\text {inj }}=10$ min and $\omega_{1}=0.2$. 

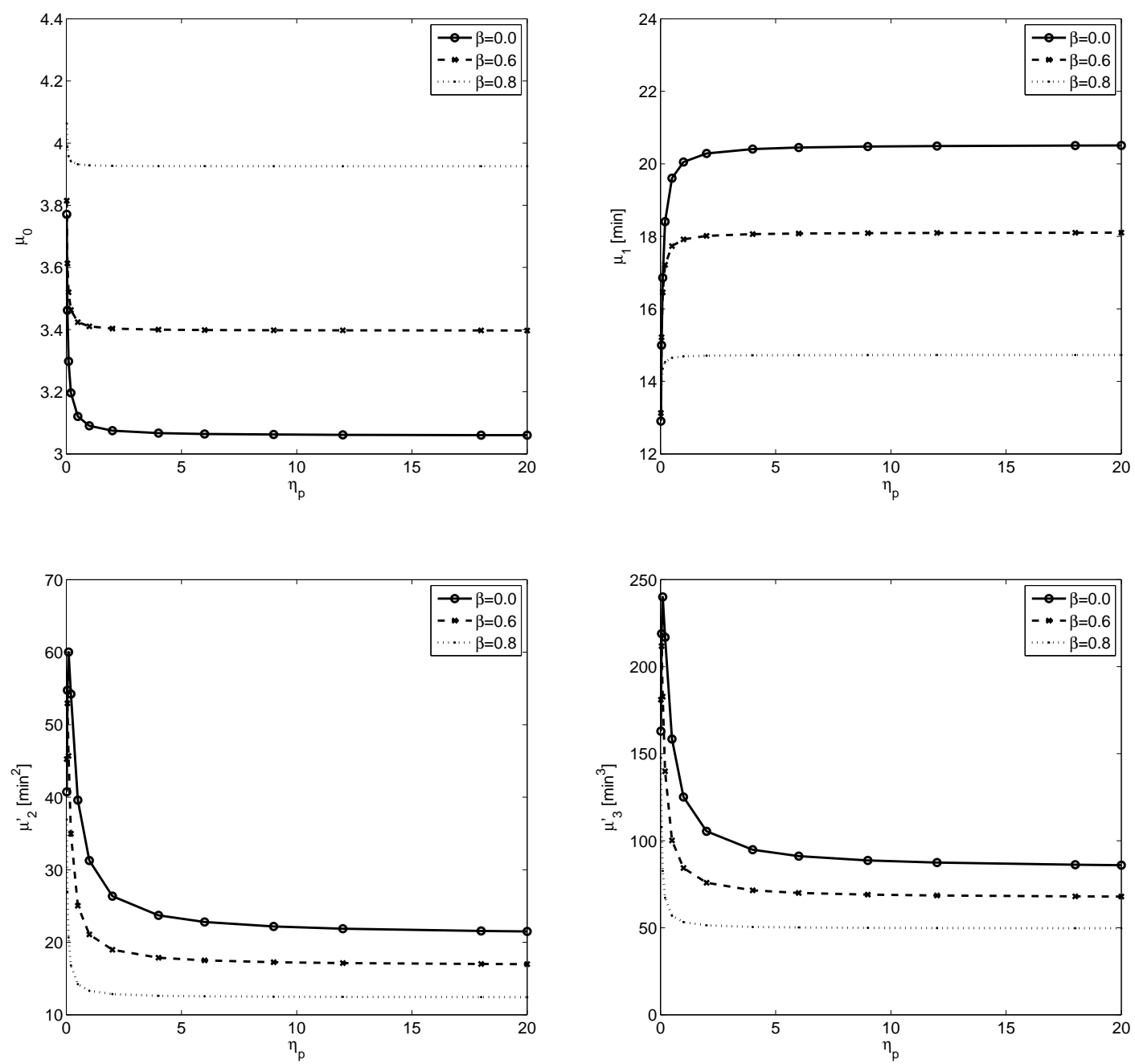

Figure 12: Irreversible reaction: Plots of average central moments (numerical) for component 1 considering different values of $\eta_{p}$ using Dirichlet BCs. Here, $c_{b_{i}, \text { inj }}=0.5 \mathrm{~g} / l(i=1,2)$, $t_{\text {inj }}=10 \mathrm{~min}$ and $\omega_{1}=0.2$. 

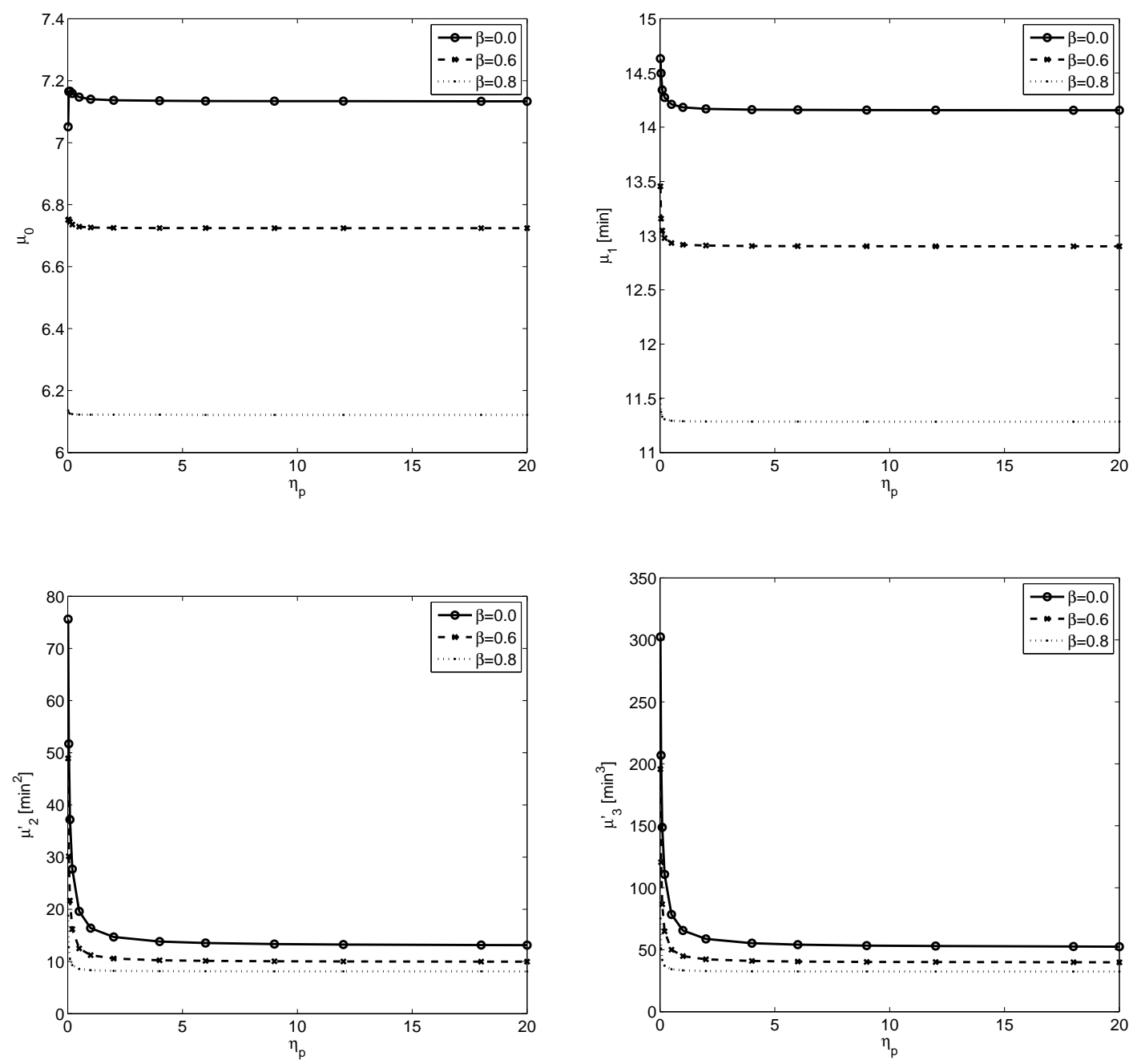

Figure 13: Irreversible reaction: Plots of average central moments (numerical) for component 2 considering different values of $\eta_{p}$ using Dirichlet BCs. Here, $c_{b_{i}, \text { inj }}=0.5 \mathrm{~g} / l(i=1,2)$, $t_{\text {inj }}=10 \mathrm{~min}$ and $\omega_{1}=0.2$. 

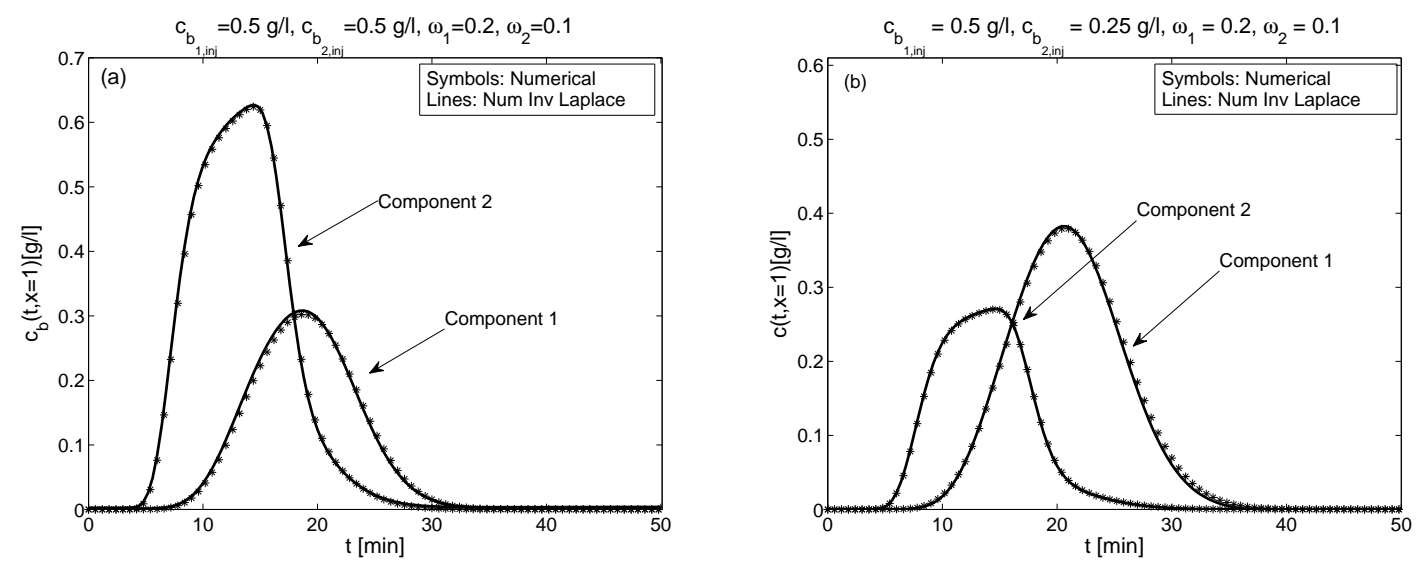

Figure 14: Reversible reaction: Influence of injection on the concentration profiles at $x=1$. Here, $c_{b_{i}, \text { inj }}=0.5 \mathrm{~g} / \mathrm{l}(i=1,2), t_{\mathrm{inj}}=10 \mathrm{~min}, \omega_{1}=0.2$ and $\omega_{2}=0.1$. 

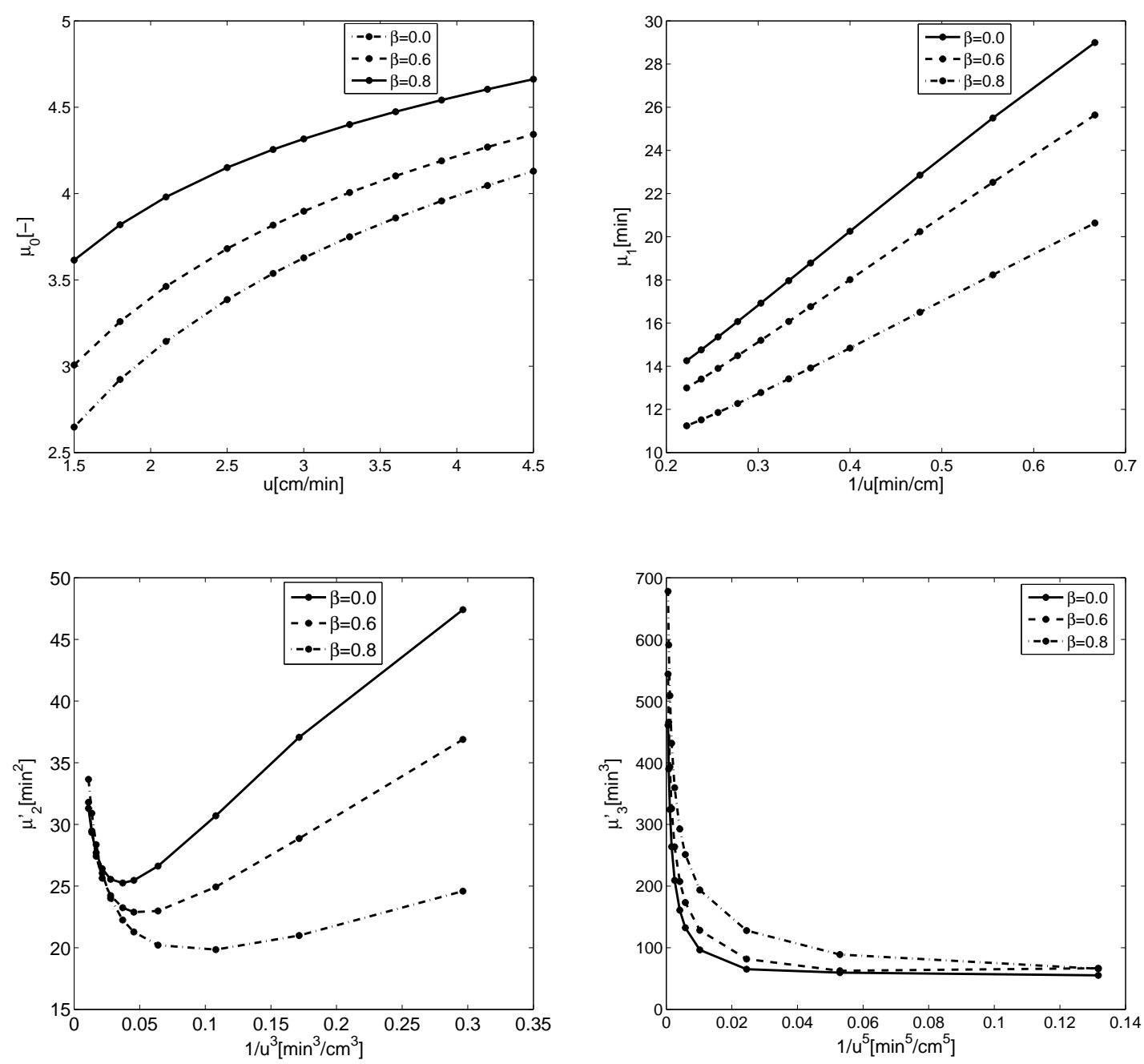

Figure 15: Reversible reaction: Plots of average central moments (numerical) of component1 considering different flow-rates using Dirichlet BCs. Here, Here, $c_{b_{i}, \text { inj }}=0.5 \mathrm{~g} / \mathrm{l}(i=$ $1,2), t_{\text {inj }}=10 \mathrm{~min}, \omega_{1}=0.2$ and $\omega_{2}=0.1$. 

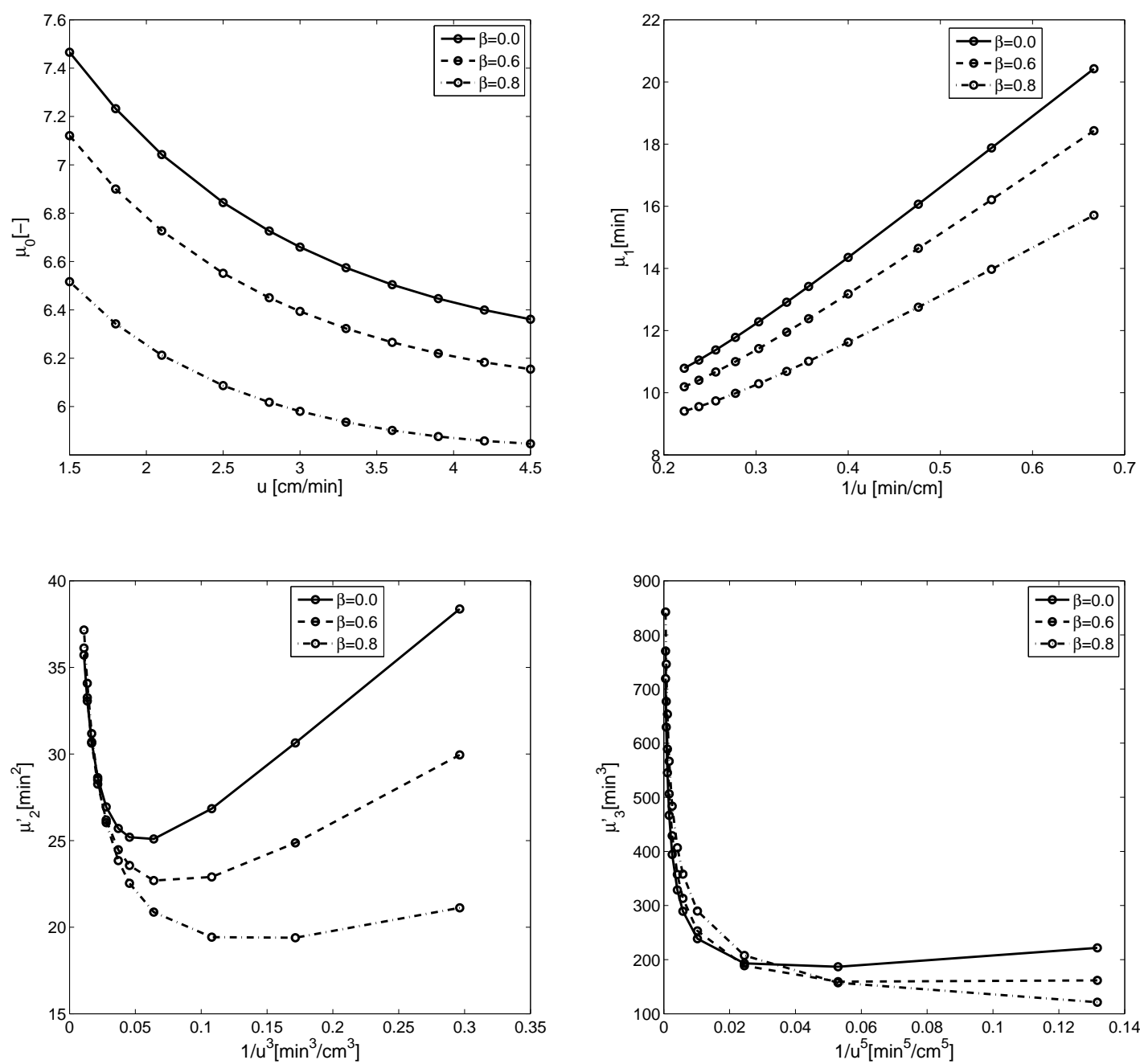

Figure 16: Reversible reaction: Plots of average central moments (numerical) of component2 considering different flow-rates using Dirichlet BCs. Here, Here, $c_{b_{i}, \text { inj }}=0.5 \mathrm{~g} / \mathrm{l}(i=$ $1,2), t_{\text {inj }}=10 \min , \omega_{1}=0.2$ and $\omega_{2}=0.1$. 


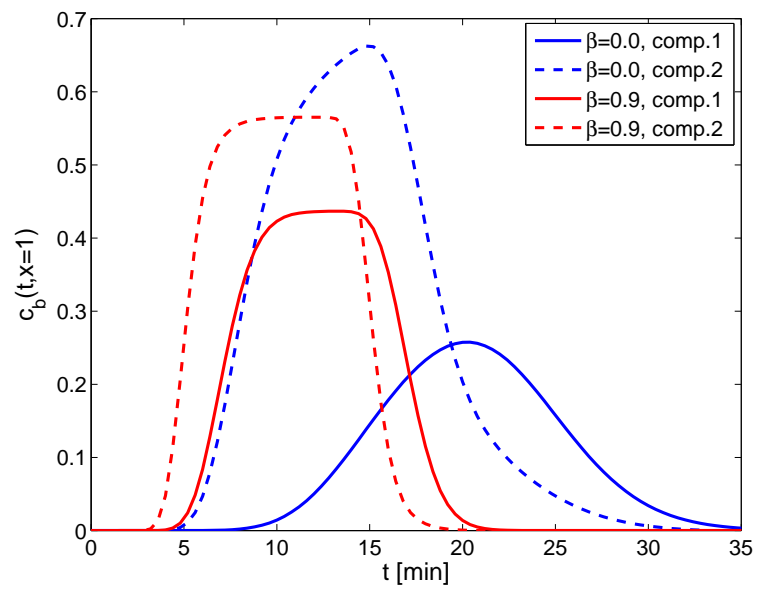

For Table of Contents only. 